\title{
Subrelations of ergodic equivalence relations $\dagger$
}

\author{
J. FELDMAN
}

Department of Mathematics, University of California, Berkeley, California 94720, USA

\author{
C. E. SUTHERLAND
}

Mathematics Department, University of New South Wales; Kensington, NSW, 2033, Australia

\section{R. J. ZIMMER}

Mathematics Department, University of Chicago, Chicago, Illinois 60637, USA

\section{(Received 29 March 1988)}

Abstract. We introduce a notion of normality for a nested pair of (ergodic) discrete measured equivalence relations of type $\mathrm{II}_{1}$. Such pairs are characterized by a group $Q$ which serves as a quotient for the pair, or by the ability to synthesize the larger relation from the smaller and an action (modulo inner automorphisms) of $Q$ on it; in the case where $Q$ is amenable, one can work with a genuine action. We classify ergodic subrelations of finite index, and arbitrary normal subrelations, of the unique amenable relation of type $\mathrm{II}_{1}$. We also give a number of rigidity results; for example, if an equivalence relation is generated by a free $\mathrm{II}_{1}$-action of a lattice in a higher rank simple connected non-compact Lie group with finite centre, the only normal ergodic subrelations are of finite index, and the only strongly normal, amenable subrelations are finite.

\section{Introduction}

The purpose of this paper is to study subrelations of ergodic equivalence relations.

Let $H \subseteq G$ be countable groups, and suppose $G$ acts as measure-preserving automorphisms on a standard space $(X, \mathscr{B}, \mu)$; we suppose the action $(x, g) \in$ $X \times G \rightarrow x g \in X$ is free, that $\mu(X)=1$ and that $H$ acts ergodically, so that if $E \in \mathscr{B}$ with $E h=E$ for all $h \in H$, then $\mu(E) \in\{0,1\}$. We let

$$
\mathscr{S}=\{(x, x g): x \in X, g \in G\} \supseteq \mathscr{R}=\{(x, x h): x \in X, h \in H\}
$$

be the equivalence relations generated by the actions of $G$ and $H$. In this context, our basic question is: to what extent does the pair $\mathscr{R} \subseteq \mathscr{S}$ 'remember' the pair $H \subseteq G$ ? Although this question is too vague to admit a precise answer, we shall see that there are circumstances in which the pair $\mathscr{R} \subseteq \mathscr{S}$ has a good memory. In particular, we shall show that if $H$ is normal in $G$, the pair $\mathscr{R} \subseteq \mathscr{S}$ determines $G / H$, and that if $H=\Gamma$ is a lattice in a higher rank (simple connected non-compact) Lie group $L$, and if $H$ is normal in $G$ with $G / H$ amenable and torsion-free, then $\mathscr{S}$ determines the rank of $L$.

† To the memory of HENRY ABEL DYE, teacher, friend and colleague. 
The proper context for our results is to consider an ergodic discrete measured equivalence relation $\mathscr{S}$ on a standard probability space $(X, \mathscr{B}, \mu)$, and to study its subrelations $\mathscr{R}$. Thus $\mathscr{S} \subseteq X \times X$ is an equivalence relation and in $\mathscr{B} \times \mathscr{B}$, the equivalence classes $\mathscr{S}(x)$ are countable, and the measure of any $\mathscr{S}$-saturated element of $\mathscr{B}$ is either 0 or 1 ; we will also assume that $\mathscr{S}$ is of type $\mathrm{II}_{1}$, a technical condition corresponding in the preceding discussion to the invariance of $\mu$ under $G$. We note that isomorphism of such equivalence relations $\mathscr{S}$ on $(X, \mathscr{B}, \mu)$, and $\mathscr{S}^{\prime}$ on $\left(X^{\prime}, \mathscr{B}^{\prime}, \mu^{\prime}\right)$ (i.e. existence of a bimeasurable map $\phi:(X, \mathscr{B}, \mu) \rightarrow\left(X^{\prime}, \mathscr{B}^{\prime}, \mu^{\prime}\right)$ with $\mu^{\prime} \circ \phi$ equivalent to $\mu$ and $\phi(\mathscr{S}(x))=\mathscr{S}^{\prime}(\phi(x))$ for $\mu$-almost all $\left.x \in X\right)$ corresponds, in the case that $\mathscr{S}$ and $\mathscr{S}^{\prime}$ are generated by free actions of countable groups $G$ and $G^{\prime}$, to H. A. Dye's notion [5], of orbit equivalence for such actions i.e. the existence of a measure space isomorphism $\phi:(X, \mathscr{B}, \mu) \rightarrow\left(X^{\prime}, \mathscr{B}^{\prime}, \mu^{\prime}\right)$ with $\phi(x G)=\phi(x) G^{\prime}$ for almost all $x \in X$. While it is known that a $\mathrm{II}_{1}$ ergodic equivalence relation generated by a free action of a lattice in a higher-rank Lie group determines that rank [12], it is also known that any two ergodic finite-measure-preserving free actions of discrete amenable groups are orbit equivalent $[5,3]$, so it might seem surprising that the pair $\mathscr{R} \subseteq \mathscr{S}$ retains any significant information about the underlying groups at all.

The plan of the paper is as follows. In $\S 1$ we show how to associate to any pair $\mathscr{R} \subseteq \mathscr{S}$ of discrete measured $\mathrm{II}_{1}$ equivalence relations, a countable index set $J$ and a cocycle $\sigma \in Z^{1}\left(S, \sum(J)\right)$, where $\sum(J)$ is the full permutation group of $J$. The cardinality of $J$ is called the index of $\mathscr{R} \subseteq \mathscr{S}$. The cocycle is called the index cocycle and depends, up to cohomology and automorphisms of $\mathscr{P}$, only on the isomorphism class of the pair $\mathscr{R} \subseteq \mathscr{S}$. We use the index cocycle to prove a 'duality' between pairs $\mathscr{R} \subseteq \mathscr{S}$ and extensions $\hat{\mathscr{R}} \stackrel{p}{\rightarrow} \mathscr{S}$ (i.e. measured discrete equivalence relations $\hat{\mathscr{R}}$ on $(Y, \nu)$ for which there is a map $p:(Y, \nu) \rightarrow(X, \mu)$ with $p(\hat{\mathscr{R}}(y))=\mathscr{R}(p(y))$ for almost all $y \in Y)$. The extension associated with $\mathscr{R} \subseteq \mathscr{S}$ is the skew product $\mathscr{S} \times{ }_{\sigma} J=\hat{\mathscr{R}}$ where $\sigma$ is the index cocycle; the pair associated to an extension $\hat{\mathscr{R}} \stackrel{p}{\rightarrow} \mathscr{S}$ is the pair $\hat{\mathscr{R}} \subseteq \hat{\mathscr{S}}$, where $\hat{\mathscr{S}}=\left\{\left(y, y^{\prime}\right):\left(p(y), p\left(y^{\prime}\right)\right) \in \mathscr{S}\right\}$. These constructions are intimately related to Jones 'basic construction', [13], in the study of sub-von-Neumann algebras; this aspect will be taken up in a subsequent paper [21].

The second section introduces the idea of a normal pair $\mathscr{R} \subseteq \mathscr{S}$ of equivalence relations. In the case of equivalence relations arising from free group actions of $H \subseteq G$ described above, it corresponds roughly to normality of $H$ in $G$. In the general case of $\mathscr{R} \subseteq \mathscr{S}$ it is defined by the triviality of the index cocycle $\sigma$ of the pair $\mathscr{R} \subseteq \mathscr{S}$ on $\mathscr{R}$, and corresponds via the duality operation, to normality (as in [26]) of the extension $\hat{\mathscr{R}} \stackrel{p}{\rightarrow} \mathscr{Y}$.

In the case where $\mathscr{R}$ is ergodic, normality of $\mathscr{R}$ in $\mathscr{S}$ may be viewed as an axiomatization of the following situation: there is a countable group $Q$ and a map $\phi: p \in Q \rightarrow \phi_{p} \in$ Aut $(\mathscr{R}, \mu)$ such that $\varepsilon \circ \phi$ is a faithful homomorphism ( $\varepsilon$ being the quotient map from Aut $(\mathscr{R}, \mu)$ to Out $(\mathscr{R}, \mu))$ and such that

$$
\mathscr{S}=\left\{\left(x, x^{\prime}\right) \in X \times X:\left(x, \phi_{p}\left(x^{\prime}\right)\right) \in \mathscr{R} \quad \text { for some } p \in Q\right\} .
$$

The group $Q$ is uniquely determined by the pair $\mathscr{R} \subseteq \mathscr{S}$ (up to isomorphism) and 
appears as the quotient, in a precise sense, of $\mathscr{S}$ by $\mathscr{R}$; in the case of actions of $H$ and $G$, with $H$ normal in $G$ as above, the quotient of $\mathscr{S}$ by $\mathscr{R}$ is precisely $G / H$ so that $\mathscr{R} \subseteq \mathscr{S}$ 'remembers' $G / H$ (even though in general $\mathscr{R} \subseteq \mathscr{S}$ need not remember $G$ or $H$ ). This is precisely the group found in [26], although the present viewpoint is a little different.

The case where $\mathscr{R}$ is normal in $\mathscr{S}$ but not ergodic is more subtle, and leads to a 'quotient groupoid' 2 rather than a group, and an 'action' of $\mathscr{Q}$ on $\mathscr{R}$ by endomorphisms rather than automorphisms. We also introduce in $\$ 2$ a notation of strong normality for a pair $\mathscr{R} \subseteq \mathscr{S}$; here we demand that $\mathscr{S}$ be generated by $\mathscr{R}$ and a countable number of automorphisms of $\mathscr{R}$. When $\mathscr{R}$ is ergodic, normality and strong normality coincide, but in general strong normality is a strictly stronger conditionsee Theorem 2.15, where we establish a link with the information cocycle of [20].

In $\S 3$, we prove some classification results. In particular we provide a complete classification of the finite index subrelations of the amenable $\mathrm{II}_{1}$ ergodic equivalence relation $\mathscr{S}$, and of its normal ergodic subrelations; the invariants are respectively conjugacy classes of transitive subgroups of finite permutation groups, and the quotient groups (which are amenable). We also prove a 'correction' result: if $Q$ is a countable amenable group and $\phi: Q \rightarrow$ Aut $(\mathscr{R})$ is a map with $\varepsilon \circ \phi$ a faithful homomorphism into Out $(\mathscr{R})$, then there is a homomorphism $\psi: Q \rightarrow$ Aut $(\mathscr{R})$ with $\varepsilon \circ \phi=\varepsilon \circ \psi$-here $\mathscr{R}$ is an ergodic $\mathrm{II}_{1}$ discrete equivalence relation which (perhaps surprisingly) is not assumed to be amenable.

In $\S 4$, we present a variety of 'rigidity type' results. In particular, we show that if $\mathscr{S}$ is generated by a free $\mathrm{II}_{1}$ action of a lattice $\Gamma$ in a simple, connected, non-compact Lie group $L$ then the only strongly normal amenable subrelations of $\mathscr{S}$ are finite, and (in the higher rank case) the only ergodic normal subrelations of $\mathscr{S}$ are of finite index. This is the ergodic-theoretic analogue of the assertion that such lattices $\Gamma$ have only finite or cofinite normal subgroups, [16]. We also show that if $\mathscr{R}$ is generated by a free ergodic $\mathrm{II}_{1}$ action of a lattice in a higher rank Lie group $L$ as above, then if $\mathscr{R}$ is normal in $\mathscr{S}$ and the quotient $Q$ is amenable and torsion free, then $\mathscr{S}$ remembers the rank of $L$. This set of results draws heavily on [24] and should be regarded as complementary to the results contained there-in.

There are many unsolved problems connected with this work - some of these are collected in the final section, with comments. We believe that most of the results may be generalized to the case where $\mathscr{R}$ and $\mathscr{S}$ are not discrete, but $\mathscr{S}$ is, in an appropriate sense 'smooth modulo $\mathscr{R}$ '. We hope to treat this in a later paper.

A note concerning the exposition. We work throughout with equivalence relations which are of type $\mathrm{II}_{1}$. At the end of each section we indicate how to extend the results to equivalence relations of other types. We hope this simplifies the exposition without detracting from the level of generality of the work.

Notation and terminology. Unless otherwise specified, $\mathscr{S}$ will denote throughout an ergodic discrete measured equivalence relation on a non-atomic standard measure space $(X, \mathscr{B}, \mu)$, of type $\mathrm{II}_{1}$, as in [10]. Thus $\mu$ is a probability measure, and there exists a countable group $G$ which acts on $(X, \mathscr{B}, \mu)$ via $(x, g) \rightarrow x g$ in such a way that $\mathscr{S}=\{(x, x g): x \in X, g \in G\}, \mu$ is invariant under $G$, and such that $G$-invariant 
measurable sets have measure 0 or $1 . \mathscr{R}$ will denote a subrelation of $\mathscr{S}$ which is also a Borel subset of $\boldsymbol{X} \times \boldsymbol{X}$, so that $\mathscr{R}$ is also a discrete measured (not necessarily ergodic) equivalence relation on $(X, \mathscr{B}, \mu)$.

If $\mathscr{R} \subseteq \mathscr{S}$ on $(X, \mathscr{B}, \mu)$ is given, and $\phi$ is a measure space automorphism of $(X, \mathscr{B}, \mu)$, we write $\phi \in$ Aut $(\mathscr{S})$ if for almost all $\left(x, x^{\prime}\right) \in X \times X,\left(x, x^{\prime}\right) \in \mathscr{S}$ if and only if $\left(\phi(x), \phi\left(x^{\prime}\right)\right) \in \mathscr{S}, \phi \in \operatorname{Int}(\mathscr{S})$ if $\phi \in \operatorname{Aut}(\mathscr{P})$ and $(\phi(x), x) \in \mathscr{S} \mu$-a.e. (so Int $(\mathscr{S})$ is the full group [ $\mathscr{S}$ ] of $\mathscr{S}$, as in [10]), and $\operatorname{Aut}_{\mathscr{S}}(\mathscr{R})=\operatorname{Aut}(\mathscr{R}) \cap \operatorname{Int}(\mathscr{S})$. We identify elements of these groups if they agree a.e. Note Int $(\mathscr{S})$ is normal in the group Aut $(\mathscr{S})$; the quotient is denoted by Out $(\mathscr{S})$. We shall also have to deal with endomorphisms $\phi$ of $(X, \mathscr{B}, \mu)$ i.e. measurable maps $\phi: X \rightarrow X$ with $\mu \circ \phi^{-1}$ equivalent to $\mu$ (in the sense of having the same null sets). End $(\mathscr{Y})$ will denote those endomorphisms $\phi$ of $(X, \mathscr{B}, \mu)$ for which $\left(x, x^{\prime}\right) \in \mathscr{S}$ implies $\left(\phi(x), \phi\left(x^{\prime}\right)\right) \in \mathscr{Y}$, and End $\operatorname{En}_{\mathscr{S}}(\mathscr{R})$ denotes those endomorphisms $\phi$ of $\mathscr{R}$ for which $(\phi x, x) \in \mathscr{S}$ a.e.

We let $\mathscr{S}(x)=\left\{x^{\prime} \in X:\left(x, x^{\prime}\right) \in \mathscr{S}\right\}$ and for $E \in \mathscr{B}, \mathscr{S}(E)=\bigcup_{x \in E} \mathscr{S}(x)$; thus $\mathscr{S}(x)$ is countable for each $x \in X$, and $\mu(\mathscr{S}(E))=0$ if $\mu(E)=0$.

Given $\mathscr{S}$ on $(X, \mathscr{B}, \mu)$, and a Polish group $A, Z^{1}(\mathscr{S}, A)$ will denote the space of measurable maps $\sigma: \mathscr{S} \rightarrow A$ for which $\sigma(x, z)=\sigma(x, y) \sigma(y, z)$ a.e. on

$$
\mathscr{S}^{(2)}=\{((x, y),(y, z)):(x, y) \in \mathscr{S},(y, z) \in \mathscr{P}\},
$$

where two such maps are identified if they agree a.e. The elements of $Z^{1}(\mathscr{S}, A)$ are called $A$-valued 1-cocycles on $\mathscr{S}$; cocycles $\sigma_{1}, \sigma_{2}$ are said to be cohomologous if there is a measurable map $\nu: X \rightarrow A$ with $\sigma_{1}(x, y)=\nu(x) \sigma_{2}(x, y) \nu(y)^{-1}$ a.e. on $\mathscr{S}$.

If $\mathscr{S}$ is given on $(X, \mathscr{B}, \mu)$, and $E \in \mathscr{B}$ with $\mu(E)>0$, we let $\left.\mathscr{Y}\right|_{E}$ denote the reduction $\mathscr{S} \cap(E \times E)$ of $\mathscr{S}$ to $E$-it again is a discrete measured ergodic $\mathrm{II}_{1}$ equivalence relation.

We shall also need to use discrete measured groupoids $(\mathscr{G}, \nu)$, as discussed in [19]; for such groupoids, $r$ and $s$ denote the range and source maps, $\mathscr{G}^{(0)}$ the space of units, and $\mathscr{G}^{(2)}$ the domain of the partially defined multiplication, so

$$
\mathscr{G}^{(2)}=\left\{\left(\gamma_{1}, \gamma_{2}\right) \in \mathscr{G} \times \mathscr{G}: r\left(\gamma_{2}\right)=s\left(\gamma_{1}\right)\right\}
$$

\section{The index cocycle and duality}

Let $\mathscr{S}$ be an ergodic, discrete, measured $\mathrm{II}_{1}$ equivalence relation on $(X, \mathscr{B}, \mu)$, and let $\mathscr{R} \subseteq \mathscr{S}$ be a Borel subrelation as in $\S 0$. Note that $\mathscr{R}$ induces an equivalence relation on each $\mathscr{S}$-class $\mathscr{S}(x)$; we let $J(x)$ denote the quotient $\mathscr{S}(x) / \mathscr{R}$.

LEMMA 1.1. (a) The cardinality $|J(x)|$ is constant $\mu$-a.e.

(b) If $N$ is the cardinal in (a), there are Borel functions $\phi_{j}: X \rightarrow X$ such that for $\mu$-a.e. $x \in X,\left\{\mathscr{R}\left(\phi_{j}(x)\right), 0 \leq j<N\right\}$ partition $\mathscr{S}(x)$.

Proof. (a) By [10, Theorem 1], we may choose Borel actions of countable groups $H$ and $G$ on $X$ which generate $\mathscr{R}$ and $\mathscr{S}$ respectively. If $h, k \in H$ and $g_{1}, \ldots, g_{n} \in G$, set

$E\left(g_{1}, \ldots, g_{n}, h, k\right)=\left\{x \in X: h g_{i} x \neq k g_{j} x \quad\right.$ for all $i, j$ with $1 \leq i, j \leq n$ and $\left.i \neq j\right\}$

$E\left(g_{1}, \ldots, g_{n}, h, k\right)$ is a Borel set and since

$$
\{x:|J(x)| \geq n\}=\bigcup_{\left(g_{1}, \ldots, g_{n}\right)} \bigcap_{h, k} E\left(g_{1}, \ldots, g_{n}, h, k\right),
$$


$x \rightarrow|J(x)|$ is Borel. To complete the proof of (a) observe that if for $z \in \mathscr{S}(x),[z]_{x}$ denotes the canonical image of $z$ in $J(x)$, then the map $\theta(x, y): J(y) \rightarrow J(x)$ defined for $(x, y) \in S$ by $\theta(x, y)\left([z]_{y}\right)=[z]_{x}$ is a bijection, so that $x \rightarrow|J(x)|$ is constant on $\mathscr{S}$-classes; since $\mathscr{S}$ is ergodic, $|J(x)|$ is constant $\mu$.a.e.

(b) Let $G=\left\{g_{n}\right\}_{n \geq 1}$ be as in the proof of (a) and define inductively $\phi_{0}(x)=x$ and $\phi_{n}(x)=g_{m}(x)$ where

$$
m=\inf \left\{k: g_{k} x \notin \bigcup_{j=0}^{n-1} \mathscr{R}\left(\phi_{j}(x)\right)\right\} .
$$

It is routine to show that the functions $\left\{\phi_{j}\right\}$ satisfy the conclusions of the lemma.

We will refer to any set of functions satisfying the conclusion of Lemma 1.1.6 as choice functions for the pair $\mathscr{R} \subseteq \mathscr{Y}$; the cardinal $N=|J(x)|$ in Lemma 1.1 will be called the index of $\mathscr{R}$ in $\mathscr{S}$-we allow infinite indices. Finally, $\sum(N)$ will denote the full permutation group on $\{0,1, \ldots, N-1\}$ for $N<\infty$ or $\{0,1,2, \ldots\}$ for $N=\infty$.

LEMMA 1.2. Let $\left\{\phi_{j}\right\}$ be choice functions for $\mathscr{R} \subseteq \mathscr{I}$, and define $\sigma: \mathscr{S} \rightarrow \sum(N)$ by $\sigma(x, y)(i)=j$ if $\mathscr{R}\left(\phi_{i}(y)\right)=\mathscr{R}\left(\phi_{j}(x)\right)$. Then

(a) $\sigma \in Z^{1}\left(\mathscr{Y}, \sum_{1}(N)\right)$;

(b) the class of $\sigma$ in $H^{1}(\mathscr{Y}, \Sigma(N))$ is independent of the choice functions $\left\{\phi_{j}\right\}$.

Proof. (a) If $(x, y),(y, z) \in \mathscr{S}$ and $\sigma(y, z)(i)=j, \sigma(x, y)(j)=k$ then

$$
\mathscr{R}\left(\phi_{k}(x)\right)=\mathscr{R}\left(\phi_{j}(y)\right)=\mathscr{R}\left(\phi_{i}(z)\right),
$$

so $\sigma(x, z)(i)=k$ and $\sigma(x, y) \sigma(y, z)=\sigma(x, z)$ as claimed.

(b) Let $\left\{\phi_{j}^{\prime}\right\}$ be another set of choice functions for $\mathscr{R} \subseteq \mathscr{S}$, and let $\sigma^{\prime}$ be the corresponding cocycle. For each $x \in X$, there is a unique $\nu_{x} \in \sum(N)$ such that $\mathscr{R}\left(\phi_{j}(x)\right)=\mathscr{R}\left(\phi_{\nu_{x}(j)}^{\prime}\right)$ for all $j$. A routine calculation shows that

$$
\sigma^{\prime}(x, y)=\nu_{x}^{-1} \circ \sigma(x, y) \circ \nu_{y^{\prime}}
$$

and (b) follows.

The cocycle $\sigma$ constructed in Lemma 1.2, or its cohomology class, will be called the index cocycle of the pair $\mathscr{R} \subseteq \mathscr{S}$. We note that any cocycle cohomologous to an index cocycle arises from a suitable selection of choice functions.

LemMA 1.3. Let $\mathscr{R} \subseteq \mathscr{S}$ on $(X, \mathscr{B}, \mu)$ be given with $\mathscr{R}$ ergodic, and let $\left\{\phi_{j}\right\}$ be choice functions for $\mathscr{R} \subseteq \mathscr{S}$. Then there are choice functions $\left\{\psi_{j}\right\}$ such that

(a) each $\psi_{j}$ is an automorphism of $(X, \mathscr{B}, \mu)$,

(b) $\left(\psi_{j}(x), \phi_{j}(x)\right) \in \mathscr{R}$ for $\mu$-a.e. $x$.

Proof. For each $j$, there is a partition $\left\{E_{k}\right\}$ of $X$ such that $\phi_{j}$ is injective on each $E_{k}$. Since

$$
1=\sum_{k} \mu\left(E_{k}\right)=\sum_{k} \mu\left(\phi_{j}\left(\left(E_{k}\right)\right),\right.
$$

there are elements $V_{k} \in \operatorname{Int}(\mathscr{R})$ such that $\left\{V_{k} \phi_{j}\left(E_{k}\right)\right\}_{k}$ partition $(X, \mu)$-see [5]. If we define $\psi_{j}$ by $\psi_{j}(x)=V_{k} \phi_{j}(x)$ for $x \in E_{k},\left\{\psi_{j}\right\}$ are the desired choice functions. 
We now turn to an important 'duality principal', which, as will be explained in [21], is the analogue in ergodic theory, of Jones 'basic construction', [13].

Let $\mathscr{R} \subseteq \mathscr{S}$ on $(X, \mu)$ be given with index cocycle $\sigma \in Z^{1}(\mathscr{Y}, \Sigma(N))$, and let $J=\{0,1, \ldots, N-1\}$ if $N<\infty$ or $\{0,1,2, \ldots\}$ if $N=\infty$.

Definition 1.4. (a) $\hat{\mathscr{R}}=\mathscr{S} \times{ }_{\sigma} J$ is the equivalence relation on $X \times J$ defined by $((x, i)(y, j)) \in \hat{\mathscr{R}}$ if and only if $(x, y) \in \mathscr{S}$ and $\sigma(x, y)(j)=i$.

(b) $\hat{\mathscr{S}}$ is the equivalence relation on $X \times J$ defined by $((x, i), y, j)) \in \hat{\mathscr{P}}$ if and only if $(x, y) \in \mathscr{S}$.

We note that $\hat{\mathscr{R}}$ is defined up to isomorphism by the pair $\mathscr{R} \subseteq \mathscr{F}$, by virtue of Lemma 1.2. Also note that if we choose an action $(x, g) \in X \times G \rightarrow x g$ of a countable group $G$ to generate $\mathscr{S}$, we may define an action of $G$ on $X \times J$ by $(x, i) g=$ $(x g, \sigma(x g, x)(i))$ which generates $\hat{\mathscr{R}}$; further, the map $p(x, i)=x$ from $X \times J$ to $X$ is equivariant for the $G$-actions, so that the system $(\hat{\mathscr{R}}, \mathscr{S}, p)$ fits into the 'extension' context of [25]. We will return to this point of view later.

The map $p$ above has the property that its restriction to each $\hat{\mathscr{R}}$ class $\hat{\mathscr{R}}(x, i)$ is a bijection of $\hat{\mathscr{R}}(x, i)$ with $\mathscr{S}(x)$; such maps have been considered in the groupoid context in [1], under the name $*$-bijections, but we will use the term class-bijective to describe this property. Evidently, if $\mathscr{S}, \mathscr{T}$ are measured equivalence relations on $(X, \mu)$ and $(Y, \lambda)$ and $q$ is a countable-to-one class-bijective homomorphism from $\mathscr{T}$ to $\mathscr{S}$, there is a Borel cocycle $\sigma \in Z^{1}\left(\mathscr{S}, \sum(N)\right)$ and an isomorphism $\theta$ of $\mathscr{T}$ with $\mathscr{S} \times{ }_{\sigma} J$ such that $p \circ \theta=q$. (Of course, $\mathscr{S} \times{ }_{\sigma} J$ exists for any cocycle $\sigma \in Z^{1}(\mathscr{P}, \Sigma(N)$ ).

Proposition 1.5. With notation as above, if $\mathscr{R} \subseteq \mathscr{S}$ is given, then

(a) there is a Borel bijection $\Phi: X \times J \rightarrow X \times J$ such that, if $\mathscr{T}(J)$ denotes the transitive relation on $J,((x, i)(y, i)) \in \hat{\mathscr{R}}$ (respectively $\hat{\mathscr{S}}$ ) if and only if $(\Phi(x, i), \Phi(y, j)) \in$ $\mathscr{R} \times \mathscr{T}(J)$ (respectively $\mathscr{S} \times \mathscr{T}(J)$ );

(b) $\mathscr{R}$ is ergodic if and only if $\hat{\mathscr{R}}$ is ergodic.

Proof. Let $\left\{\phi_{j}\right\}$ be choice functions for $\mathscr{R} \subseteq \mathscr{S}$ with each $\phi_{j}$ bijective, and define $\Phi: X \times J \rightarrow X \times J$ by $\Phi(x, j)=\left(\phi_{j}(x), j\right)$; the properties ascribed to $\Phi$ in (a) are routine to verify, and $\Phi$ is evidently bijective since each of the functions $\phi_{j}$ are, yielding (a). The assertion (b) is now trivial.

TheOREM 1.6. Let $\mathscr{S}$ on $(X, \mu)$ be given. Then

(a) if $\sigma \in Z^{1}\left(\mathscr{S}, \sum(J)\right)$ is such that $S \times{ }_{\sigma} J$ is ergodic, $\sigma$ is the index cocycle of some ergodic subrelation $\mathscr{R}$ of $\mathscr{Y}$;

(b) if $\mathscr{R}, \mathscr{R}^{\prime} \subseteq \mathscr{S}$ are ergodic and have index cocycles $\sigma \in Z^{1}(\mathscr{S}, \Sigma(J))$, $\sigma^{\prime} \in$ $Z^{1}\left(\mathscr{S}, \sum\left(J^{\prime}\right)\right.$ ) then $\mathscr{R}$ and $\mathscr{R}^{\prime}$ are conjugate under Aut $(\mathscr{S})$ (respectively Int $(\mathscr{S})$ ) if and only if $J=J^{\prime}$ and $[\sigma]=\left[\sigma^{\prime}\right]$ up to Aut $(\mathscr{S})$ (respectively $[\sigma]=\left[\sigma^{\prime}\right]$ ).

Proof. (a) Let $\sigma \in Z^{1}(\mathscr{P}, \Sigma(J))$ be ergodic, and let

$$
\mathscr{R}=\{(x, y) \in \mathscr{S}: \sigma(x, y)(0)=0\} .
$$

Since $\mathscr{S} \times{ }_{\sigma} J$ is ergodic, we may choose Borel functions $\phi_{j}: X \rightarrow X, j \in J$ with $\left(x, \phi_{j}(x)\right) \in \mathscr{S}$ and $\sigma\left(x, \phi_{j}(x)\right)(0)=j$. We claim that $\left\{\phi_{j}\right\}$ are choice functions for $\mathscr{R} \subseteq \mathscr{Y}$. For if $(x, y) \in \mathscr{S}$ and $\sigma(x, y)(0)=j$, then $\sigma\left(\phi_{j}(x), y\right)(0)=0$ and so $\left(\phi_{j}(x), y\right) \in$ $\mathscr{R}$; also if $z \in \mathscr{R}\left(\phi_{j}(x)\right) \cap \mathscr{R}\left(\phi_{k}(x)\right)$, then $\sigma(z, x)(j)=0$ and $\sigma(z, x)(k)=0$, so that 
$j=k$. Finally $\mathscr{R}\left(\phi_{j}(x)\right)=\mathscr{R}\left(\phi_{k}(y)\right)$ if and only if $\sigma\left(\phi_{j}(x), \phi_{k}(y)\right)(0)=0$, and hence if and only if $\sigma(x, y)(k)=j$, since

$$
\sigma(x, y)=\sigma\left(x, \phi_{j}(x)\right) \sigma\left(\phi_{j}(x), \phi_{k}(y)\right) \sigma\left(\phi_{k}(y), y\right) .
$$

Thus the index cocycle for $\mathscr{R} \subseteq \mathscr{S}$ defined by $\left\{\phi_{j}\right\}$ is precisely $\sigma$.

(b) Let $\mathscr{R}, \mathscr{R}^{\prime} \subseteq \mathscr{S}$ be given, and suppose $\left\{\phi_{j}\right\},\left\{\phi_{j}^{\prime}\right\}$ are (bijective) choice functions for $\mathscr{R}$ and $\mathscr{R}^{\prime}$. If $\theta \in$ Aut $(\mathscr{S})$ and $\theta(\mathscr{R})=\mathscr{R}^{\prime}$, then for each $x, \mathscr{R}^{\prime}(x)=\theta \mathscr{R}\left(\theta^{-1} x\right)$, so that we may choose $\phi_{j}^{\prime}(x)=\theta \circ \phi_{j} \circ \theta^{-1}(x)$; a straightforward calculation shows that with these choice functions $\sigma^{\prime}(\theta(x), \theta(y))=\sigma(x, y)$. Since, if $\theta$ is inner (i.e. $\theta \in[\mathscr{Y}]$ ), $\sigma^{\prime} \circ(\theta \times \theta)$ is cohomologous to $\sigma^{\prime}$, we obtain one of the desired implications. Conversely, suppose $\sigma^{\prime} \circ(\theta \times \theta)$ is cohomologous to $\sigma$. Replacing $\mathscr{R}^{\prime}$ by $\theta^{-1}\left(\mathscr{R}^{\prime}\right)$, we may suppose that $\sigma^{\prime}$ is cohomologous to $\sigma$; changing the choice functions for either $\mathscr{R}$ or $\mathscr{R}^{\prime}$ we may assume that $\sigma=\sigma^{\prime}$. We now have $\mathscr{S} \times{ }_{\sigma} J=S \times_{\sigma^{\prime}} J$; by Proposition 1.5(c), the map

$$
(x, j) \in X \times J \rightarrow\left(\phi_{j}^{-1} \circ \phi_{j}^{\prime}(x), j\right)
$$

gives an isomorphism of $\mathscr{R}^{\prime} \times \mathscr{T}(J)$ with $\mathscr{R} \times \mathscr{T}(J)$. Since

$$
\left(\phi_{j}^{-1} \circ \phi_{j}^{\prime}(x), x\right) \in \mathscr{S} \quad \mu . \text { a.e. in } x,
$$

any of the maps $\theta_{j}=\phi_{j}^{-1} \circ \phi_{j}^{\prime}$ provides an inner automorphism of $\mathscr{S}$ which carries $\mathscr{R}^{\prime}$ to $\mathscr{R}$ as required.

For completeness we record the following.

Proposition 1.7. Let $\mathscr{R} \subseteq \mathscr{S}$ have index cocycle $\sigma \in Z^{1}\left(\mathscr{S}, \sum(J)\right)$. Then $\sigma$ cobounds if and only if there is a partition $\left\{X_{j}\right\}_{j=0}^{N-1}$ of $X$ such that $\mu\left(X_{j}\right)>0, \mathscr{R}\left(X_{j}\right)=X_{j}$ and $\left.\mathscr{R}\right|_{X_{j}}=\left.\mathscr{P}\right|_{X_{j}}$ for each $j \in J$.

Proof. Suppose $X=\bigcup_{j=0}^{N-1} X_{j}$ with $X_{j} \cap X_{k}=\phi, \mu\left(X_{j}\right)>0, \quad \mathscr{R}\left(X_{j}\right)=X_{j}$ and $\left.\mathscr{S}\right|_{X_{j}}=\left.\mathscr{R}\right|_{X_{j}}$ for each $j$. By ergodicity of $\mathscr{S}$ we may choose Borel maps $\phi_{j}: X \rightarrow X_{j}$ with $\left\{\mathscr{R}\left(\phi_{j}(x)\right)\right\}_{j}$ partitioning $\mathscr{S}(x)$ for each $x$. The cocycle $\sigma$ defined by the choice maps $\left\{\phi_{j}\right\}$ is easily seen to be the identity.

Conversely if $\mathscr{R} \subseteq \mathscr{S}$ has trivial index cocycle, there are choice maps $\left\{\phi_{j}\right\}$ such that for $(x, y) \in \mathscr{P}, \mathscr{R}\left(\phi_{i}(x)\right)=\mathscr{R}\left(\phi_{j}(y)\right)$ if and only if $i=j$. With $X_{j}=\mathscr{R}\left(\phi_{j}(X)\right)$, it is easily checked that $\left\{X_{j}\right\}$ partition $X$, and $\left.\mathscr{R}\right|_{X_{j}}=\left.\mathscr{S}\right|_{X_{j}}$ for all $j$.

We note in particular that if $\mathscr{R} \subseteq \mathscr{S}$ is ergodic, the index cocycle for the pair $\mathscr{R} \subseteq \mathscr{S}$ does not cobound.

We take the opportunity to correct an error in [22, Theorem 5.2, p. 1082] where it is asserted that ' $\mathscr{H}_{n}=\sigma^{-1}\left(H_{n}\right)$ has finite equivalence classes'. The correct assertion is that $\mathscr{H}_{n}$ is smooth - we conclude that $\mathscr{H}=\bigcup_{n=1}^{\infty} \mathscr{H}_{n}$ is a union of smooth equivalence relations, and hence hyperfinite, so the conclusion of [22, Theorem 5.2] remains true. The smoothness is justified by the following result.

LEMMA 1.8. Let $\mathscr{R}=\Delta X \subseteq \mathscr{S}$ have choice functions $\left\{\phi_{j}\right\}$ and associated cocycle $\sigma$, and let

$$
H_{n}=\left\{\pi \in \sum(N): \pi(i)=i \text { for all } i>n\right\}
$$

Then $\sigma^{-1}\left(H_{n}\right)$ is smooth. 
Proof. Note $(x, y) \in \sigma^{-1}\left(H_{n}\right)$ if and only if $\phi_{i}(x)=\phi_{i}(y)$ for all $i>n$. Thus $\left\{\phi_{i}: i>n\right\}$ are a countable family of $\sigma^{-1}\left(H_{n}\right)$-invariant Borel functions which distinguish $\sigma^{-1}\left(H_{n}\right)$-equivalence classes. By [14, Theorem 6.2], $\sigma^{-1}\left(H_{n}\right)$ is smooth.

Finally some comments on the case where $\mathscr{S}$ on $(X, \mathscr{B}, \mu)$ is not assumed to be of type $\mathrm{II}_{1}$. Lemma 1.1, 1.2 and Proposition 1.7 remain true as stated; Lemma 1.3 is true if $\mathscr{S}$ (and hence $\mathscr{R}$ ) admits any invariant measure equivalent to $\mu$ (i.e. $\mathscr{S}$ is of type II), or if $\mathscr{R}$ admits no invariant measure equivalent to $\mu$ (i.e. $\mathscr{R}$ is of type III). The proofs in these cases are routine, but we do not know if the Lemma remains true in the remaining case ( $\mathscr{R}$ of type II, and $\mathscr{S}$ of type III). Proposition 1.5 and Theorem 1.6 hold as stated whenever Lemma 1.3 holds, with the same proof; in fact 1.5(a) holds in general with the word 'bijective' replaced by 'map', and 1.5(b) and 1.6(a) hold as stated in the general case.

\section{Normality}

We now isolate a distinguished class of subrelations, the normal ones. We keep the notation of $\S 1$.

Definition 2.1. Let $\mathscr{R} \subseteq \mathscr{S}$ have index cocycle $\sigma$. Then $\mathscr{R}$ is normal in $\mathscr{S}$ if the restriction $\left.\sigma\right|_{\mathscr{R}}$ of $\sigma$ to $\mathscr{R}$ cobounds.

We note that any finite (or indeed any smooth) subrelation of $\mathscr{S}$ is normal, since any such relation has trivial first cohomology.

TheOREM 2.2. Let $\mathscr{R} \subseteq \mathscr{S}$ be given, with index cocycle $\sigma$. Then the following are equivalent.

(a) $\mathscr{R}$ is normal in $\mathscr{S}$;

(b) there are choice functions $\left\{\phi_{j}\right\}$ for $\mathscr{R} \subseteq \mathscr{S}$ with $\phi_{j} \in \operatorname{End}_{\mathscr{S}}(\mathscr{R})$ for all $j$;

(c) the extension p: $\hat{\mathscr{R}}=\mathscr{S} \times_{\sigma} J \rightarrow \mathscr{S}$ is normal in the sense of Zimmer, [25];

(d) there is a discrete, ergodic measured groupoid $(2, \nu)$ and a homomorphism $\theta: \mathscr{P} \rightarrow \mathscr{Q}$ such that

(i) $\operatorname{ker} \theta=\mathscr{R}$

(ii) for any $\gamma \in \mathscr{Q}$ and $x \in X$ with $\theta(x)=s(\gamma)$, the source of $\gamma$, there exists $y \in X$ with $(y, x) \in \mathscr{S}$ and $\theta(y, x)=\gamma$;

(iii) for any discrete ergodic measured groupoid $\left(2^{\prime}, \nu^{\prime}\right)$ and homomorphism $\theta^{\prime}: \mathscr{S} \rightarrow \mathscr{Q}^{\prime}$ with $\operatorname{ker} \theta^{\prime} \supseteq \mathscr{R}$ there is a homomorphism $\kappa: \mathscr{Q} \rightarrow \mathscr{Q}^{\prime}$ with $\kappa \circ \theta=\theta^{\prime}$.

Before we begin the proof, some comments are in order. By normality in the sense of Zimmer, we mean the following adaptation of [25, Definition 5.4].

Definition 2.3. If $\mathscr{S}$ on $(X, \mu)$ and $\sigma \in Z^{1}\left(\mathscr{S}, \sum(N)\right)$ are given, and $u_{\sigma}(x, y): \ell^{2}(J) \rightarrow$ $\ell^{2}(J)$ is the unitary cocycle defined by $\left(u_{\sigma}(x, y) \xi\right)(j)=\xi\left(\sigma(x, y)^{-1}(j)\right)$ for $\xi \in \ell^{2}(J)$, we say $\sigma$ is normal or the extension $p: \mathscr{S} \times{ }_{\sigma} J \rightarrow \mathscr{S}$ is normal, if

$$
u_{\sigma} \circ(p \times p) \in Z^{1}\left(\mathscr{S} \times{ }_{\sigma} J ; U\left(\ell^{2}(J)\right)\right)
$$

cobounds. 
We note that this is equivalent to [25, Definition 5.4] in the case where $\mathscr{P}$ is generated by an action of a countable group $G$, and we view $\mathscr{S} \times{ }_{\sigma} J$ as being generated by the action $G$ described in the discussion following Definition 1.4.

The property ascribed to $\theta$ in Theorem 2.2(d)(ii) will be referred to as classsurjectivity. In view of 2.2 (d)(iii), the groupoid $(2, \nu)$ is unique up to isomorphism and will be referred to as the quotient $\mathscr{S} \mid \mathscr{R}$ of $\mathscr{S}$ by $\mathscr{R}$.

\section{Proof of Theorem 2.2.}

(a) $\Leftrightarrow$ (b). If $\left.\sigma\right|_{\mathscr{R}}$ cobounds, we may select choice functions $\left\{\phi_{j}\right\}$ for $\mathscr{R} \subseteq \mathscr{S}$ such that if $\sigma$ is the associated cocycle, $\sigma(x, y)=1$ for $(x, y) \in \mathscr{R}$. But now, for $(x, y) \in \mathscr{R}$, $\sigma(x, y)(i)=i$ so that $\mathscr{R}\left(\phi_{i}(x)\right)=\mathscr{R}\left(\phi_{i}(y)\right)$, or $\left(\phi_{i}(x), \phi_{i}(y)\right) \in \mathscr{R}$ for all $i$. Conversely if $\left\{\phi_{i}\right\} \in \operatorname{Aut}_{\mathscr{S}}(\mathscr{R})$ are choice functions, the associated cocycle $\sigma$ clearly satisfies $\sigma(x, y)=1$ for $(x, y) \in \mathscr{R}$.

(a) $\Leftrightarrow$ (c). Suppose that $\sigma(x, y)=1$ for $(x, y) \in \mathscr{R}$. Now $u_{\sigma}(x, y)=1$ for $(x, y) \in \mathscr{R}$. For each $x \in X$, choose $i_{0}(x) \in J$ such that $\left(x, \phi_{i_{0}(x)}(x)\right) \in \mathscr{R}$ and note that for $\gamma=$ $((x, j)(y, k)) \in \mathscr{S} \times{ }_{\sigma} J$, we have $\gamma=\gamma_{1}(x, j) \gamma_{2} \gamma_{1}(y, k)^{-1}$, where

$$
\gamma_{1}(x, j)=\left((x, j),\left(\phi_{j}(x), i_{0}\left(\phi_{j}(x)\right)\right)\right.
$$

and

$$
\gamma_{2}=\left(\phi_{j}(x), i_{0}\left(\phi_{j}(x)\right)\right),\left(\phi_{k}(y), i_{0}\left(\phi_{k}(y)\right)\right) .
$$

Since $\left(\phi_{j}(x), \phi_{k}(y)\right) \in \mathscr{R}$ and $\sigma=1$ on $\mathscr{R}$ we have

$$
u_{\sigma}(x, y)=u_{\sigma}\left(\gamma_{1}(x, j)\right) u_{\sigma}\left(\gamma_{1}(y, k)\right)^{-1}
$$

so that $u_{\sigma} \circ(p \times p)$ cobounds, as required.

Conversely, suppose $u_{\sigma} \circ(p \times p)$ cobounds; from the factorization $\gamma=$ $\gamma_{1}(x, j) \gamma_{2} \gamma_{1}(y, k)^{-1}$ above, we see that the cocycle $(x, y) \in \mathscr{R} \rightarrow u_{\sigma}(x, y)$ also cobounds. So suppose $u_{\sigma}(x, y)=v_{1}(x)^{-1} v_{2}(y)$ for $(x, y) \in \mathscr{R}$ where $x \rightarrow v_{1}(x)$ is a Borel family of unitaries on $\ell^{2}(J)$. Observe that if $\mathscr{A}=\ell^{\infty}(J)$ acting on $\ell^{2}(J)$, Ad $u_{\sigma}(x, y)(\mathscr{A})=\mathscr{A}$, so $\operatorname{Ad} v_{1}(y)(\mathscr{A})=\operatorname{Ad} v_{1}(x)(\mathscr{A})$ for $(x, y) \in \mathscr{R}$. We may thus choose a Borel family of unitaries $x \rightarrow w(x)$ on $\ell^{2}(J)$, constant on $\mathscr{R}$-classes, such that if $v_{2}(x)=w(x) v_{1}(x)$, Ad $v_{2}(x)(\mathscr{A})=\mathscr{A}$ and $u_{\sigma}(x, y)=v_{2}(x)^{-1} v_{2}(y)$ for $(x, y) \in \mathscr{R}$. Now each of the unitaries $v_{2}(x)$ is of the form $v_{2}(x)=M(x) v_{3}(x)$, where $M(x)$ is a multiplication operator and $v_{3}(x)$ is of the form $\left(v_{3}(x) \xi\right)(j)=\xi\left(\nu_{x}(j)\right)$ for some permutation $\nu_{x}$ of $J$; it follows that $\sigma(x, y)^{-1}=\nu_{y} \nu_{x}^{-1}$ so that $\sigma$ also cobounds on $\mathscr{R}$. (The argument above is adapted from $[9$, p. 178]).

(b) $\Leftrightarrow$ (d) We suppose first that there is a discrete measured ergodic groupoid $(\mathscr{Q}, \nu)$ and a homomorphism $\theta: \mathscr{S} \rightarrow \mathscr{Q}$ with the properties given in (d). Since $(\mathscr{Q}, \nu)$ is ergodic and discrete, the sets $\left\{\gamma \in \mathscr{Q}: r(\gamma)=y \in \mathscr{Q}^{(0)}\right\}$ have constant cardinality, so that there is a Borel isomorphism $\kappa: \mathscr{Q} \rightarrow Y \times J$ with $q \circ \kappa=\kappa \circ s$, where $q$ is the projection on $Y$ - here $Y=\mathscr{G}^{(0)}$ and $J=\{0,1,2, \ldots, N-1\}$ or $\{0,1,2, \ldots\}$ as usual. In view of property (d)(ii), and using a routine selection argument we may choose Borel maps $\phi_{j}: X \rightarrow X$ such that for each $x \in X, \kappa \circ \theta\left(\phi_{j}(x), x\right)=(\kappa(\theta(x)), j)$. Note that if $\left(x, x^{\prime}\right) \in \mathscr{R}, \theta(x)=\theta\left(x^{\prime}\right)$ so that $\kappa \circ \theta\left(\phi_{j}(x), x\right)=\kappa \circ \theta\left(\phi_{j}\left(x^{\prime}\right), x^{\prime}\right)$ and hence (since $\theta\left(x, x^{\prime}\right)$ is a unit 2) $\theta\left(\phi_{j}(x), \phi_{j}\left(x^{\prime}\right)\right)$ is also a unit in 2 . Since $\operatorname{ker} \theta=\mathscr{R}$ we 
conclude that $\phi_{j} \in \operatorname{End}_{\mathscr{S}}(\mathscr{R})$. To see that $\left\{\phi_{j}\right\}$ are choice functions for $\mathscr{R} \subseteq \mathscr{Y}$, note that if $(z, x) \in \mathscr{S}$ and $\kappa \circ \theta(z, x)=(y, j)$, then $\theta(z, x)=\theta\left(\phi_{j}(x), x\right)$ so that $\left(z, \phi_{j}(x)\right) \in$ $\mathscr{R}$; it follows that $\left\{\mathscr{R}\left(\phi_{j}(x)\right\}\right.$ partition $\mathscr{S}(x)$, as required.

We note that the argument above in fact shows that the kernel of any classsurjective homomorphism from $\mathscr{S}$ to a discrete ergodic measured groupoid is normal in $\mathscr{S}$.

We now turn to the construction of the quotient groupoid $\mathscr{S} \mathscr{R}$ in the case $\mathscr{R}$ is normal in $\mathscr{S}$. Choose a family $\left\{\phi_{j}\right\}$ of choice functions for $\mathscr{R} \subseteq \mathscr{S}$ with $\phi_{j} \in \operatorname{End}_{\mathscr{S}}(R)$. We let $\mu=\int_{Y}^{\oplus} \mu_{y} d \nu(y)$ be the ergodic decomposition of $\mu$ with respect to $\mathscr{R}$. We will produce a groupoid structure on $J \times Y$; of course if $\mathscr{R}$ is ergodic, $Y$ is just a point, and our groupoid will in fact be a group. For $j, k \in J$ and $x \in X$, define $j *_{x} k=\ell$ to mean that $\mathscr{R}\left(\phi_{j}\left(\phi_{k}(x)\right)=\mathscr{R}\left(\phi_{\ell}(x)\right)\right.$. Since $\phi_{j} \in \operatorname{End}_{\mathscr{S}}(\mathscr{R})$ for all $j, j *_{x} k$ is constant on $\mathscr{R}$-classes, so there is a well defined Borel map $(j, y, k) \in J \times Y \times J \rightarrow j *_{y} k \in J$ satisfying $j *_{\alpha(x)} k=j *_{x} k$, where $\alpha: X \rightarrow Y$ is the canonical projection. In addition, since $x \rightarrow \alpha\left(\phi_{j}(x)\right)$ is constant on $\mathscr{R}$-classes for each $j \in J$, there is a Borel map $\Phi_{j}: Y \rightarrow Y$ such that $\Phi_{j} \circ \alpha=\alpha \circ \phi_{j}$.

We now define a groupoid structure 2 on $J \times Y$ by

$$
\mathscr{Q}^{(2)}=\left\{\left(k, \Phi_{j}(y)\right)(j, y): k, j \in J, y \in Y\right\}
$$

with product

$$
\left(k, \Phi_{j}(y)\right)(j, y)=(k \underset{y}{* j}, y) .
$$

We will check shortly that $\mathscr{Q}$ is indeed a groupoid with unit space (isomorphic to) $Y$, and that $(2, \nu)$ is a discrete measured ergodic groupoid, but first we introduce the 'homomorphism' $\theta$ and establish some of its properties. We define

$$
\theta\left(x^{\prime}, x\right)=(j, \alpha(x)) \quad \text { if } \quad\left(x^{\prime}, \phi_{j}(x)\right) \in \mathscr{R} .
$$

The class-surjectivity follows from the observation that $\theta\left(\phi_{j}(x), x\right)=(j, \alpha(x))$ for each $j$. Secondly, if

$$
\left(x^{\prime \prime}, x^{\prime}\right) \in \mathscr{S},\left(x^{\prime}, x\right) \in \mathscr{S} \text { and } \theta\left(x^{\prime \prime}, x^{\prime}\right)=\left(k, \alpha\left(x^{\prime}\right)\right), \theta\left(x^{\prime}, x\right)=(j, \alpha(x)),
$$

than the product $\left(k, \alpha\left(x^{\prime}\right)\right)(j, \alpha(x))$ is defined in 2 and equal to $\left(k *_{x} j, \alpha(x)\right)$. However

$$
x^{\prime \prime} \sim_{\mathscr{R}} \phi_{k}\left(x^{\prime}\right) \sim_{\mathscr{R}} \phi_{k}\left(\phi_{j}(x)\right) \sim_{\mathscr{R}} \phi_{\ell}(x) \quad \text { where } \ell=k_{x}^{* j} j
$$

thus $\theta\left(x^{\prime \prime}, x\right)=\left(k *_{x} j, \alpha(x)\right)$ and $\theta$ is multiplicative. To see 2 is a groupoid, we need

LEMMA 2.3. The product in 2 is associative, and a Borel map from $\mathscr{Q}^{(2)}$ to 2 .

Proof. Suppose that $x, y, z \in X$, and that the product $(j, \alpha(x))((k, \alpha(y))(\ell, \alpha(z))$ is defined in 2. Then

$$
\alpha(y)=\Phi_{\ell}(\alpha(z)), \quad \alpha(x)=\Phi_{m}(\alpha(z)) \quad \text { where } m=k \underset{\alpha(z)}{*} \ell,
$$

and the triple product is $\left(j *_{\alpha(z)}\left(k *_{\alpha(z)} \ell, \alpha(z)\right)\right.$.

A routine calculation shows that $\Phi_{m}(\alpha(z))=\Phi_{k}\left(\Phi_{\ell}(\alpha(z))\right)$, so the product $(j, \alpha(x))(k, \alpha(y))$ is defined in $\mathscr{Q}$ and equal to $\left(j *_{\alpha(y)} k, \Phi_{\ell}(\alpha(z))\right)$. Thus the triple 
product $((j, \alpha(x))(k, \alpha(y)))(\ell, \alpha(z))$ is defined and equal to $\left(\left(j *_{\alpha(y)} k\right) *_{\alpha(z)} \ell, \alpha(z)\right)$. It thus suffices to show

$$
(j \underset{\alpha(y)}{*} k) \underset{\alpha(z)}{*} \ell=j \underset{\alpha(z)}{*}(k \underset{\alpha(z)}{*} \ell) .
$$

But $j *_{\alpha(z)}\left(k *_{\alpha(z)} \ell\right)=n$ if and only if

$$
\mathscr{R}\left(\left(\phi_{n}(z)\right)=\mathscr{R}\left(\phi_{j}\left(\phi_{k} \circ \phi_{\ell}(z)\right)\right)\right. \text {, }
$$

and $\left(j *_{\alpha(y)} k\right) *_{\alpha(z)} \ell=n$ if and only if $\mathscr{R}\left(\phi_{j}^{\circ} \phi_{k}\left(\phi_{\ell}(z)\right)\right)=\mathscr{R}\left(\phi_{n}(z)\right)$; associativity is thus established. The fact that the product map and its domain in $2 \times 2$ are Borel trivial.

Leмmа 2.4. For all $\gamma \in \mathscr{Q}$, there are unique elements $u, v \in \mathscr{Q}$, with $u \gamma=\gamma=\gamma v$.

Proof. For each $x \in X$, there is a unique $i_{0}(x) \in J$ such that $\mathscr{R}\left(\phi_{i_{0}(x)}(x)\right)=\mathscr{R}(x)$. Observe that $i_{0}$ is Borel and constant on $\mathscr{R}$-class, so that we may view $i_{0}$ as being defined on $Y$. It is now routine to verify that $\gamma=(k, y) \in \mathscr{Q}$, then $u=$ $\left(i_{0}\left(\Phi_{k}(y)\right), \Phi_{k}(y)\right)$ and $v=\left(i_{0}(y), y\right)$ are the desired left and right units.

It is now evident that the unit space of $\mathscr{2}$ can be identified with $Y$.

Lemma 2.5. 2 admits inverses, and $\gamma \rightarrow \gamma^{-1}$ is Borel on 2.

Proof. Given $(k, x) \in J \times X$, there is a unique $\ell=\ell(k, x)$ with $\mathscr{R}\left(\phi_{\ell}\left(\phi_{k}(x)\right)=\mathscr{R}(x)\right.$; further, $\ell(k, x)$ is Borel and, for each $k$, constant on $\mathscr{R}$-classes, so we may view $\ell$ as being defined on $J \times Y$. For $(k, y) \in \mathscr{Q}$ we have

$$
\left(\ell(k, y), \Phi_{k}(y)\right)(k, y)=\left(i_{0}(y), y\right)
$$

trivially, while

$$
(k, y)\left(\ell(k, y), \Phi_{k}(y)\right)=\left(i_{0}\left(\Phi_{k}(y)\right), \Phi_{k}(y)\right)
$$

since $\mathscr{R}\left(\phi_{k} \circ \phi_{\ell(k, x)}\left(\phi_{k}(x)\right)=\mathscr{R}\left(\phi_{k}(x)\right)\right.$, which implies that with $z=\Phi_{k}(y)$,

$$
k *_{z} \ell(k, y)=i_{0}\left(\Phi_{k}(y)\right) \text {. }
$$

Thus $(k, y)^{-1}=\left(\ell(k, y), \Phi_{k}(y)\right)$; evidently, $(k, y) \rightarrow(k, y)^{-1}$ is Borel.

We now return to the proof of Theorem 2.2. First, note that if $E \subseteq Y$ is $\nu$-null, then $\alpha^{-1}(E)$, and hence $\phi_{k}\left(\alpha^{-1}(E)\right)$ and $\mathscr{R}\left(\phi_{k}\left(\alpha^{-1}(E)\right)\right)$, are $\mu$-null, so that if $F$ is the 2 -saturation of $E, \nu(F)=\mu\left(\bigcup_{k} \mathscr{R}\left(\phi_{k}\left(\alpha^{-1} E\right)\right)\right)$ is also zero. The ergodicity of $(2, \nu)$ follows automatically from that of $\mathscr{S}$ on $(X, \mu)$. To determine ker $\theta$, let $\left(x^{\prime}, x\right) \in \mathscr{S}$, and observe that $\theta\left(x^{\prime}, x\right)=\left(i_{0}(\alpha(x)), \alpha(x)\right)$ is a unit if and only if $\left(x^{\prime}, \phi_{i_{0}(x)}(x)\right) \in \mathscr{R}$ and hence if and only $\left(x^{\prime}, x\right) \in \mathscr{R}$, by the choice of $i_{0}(x)$.

Finally, suppose $\theta^{\prime}: \mathscr{S} \rightarrow \mathscr{Q}^{\prime}$ is a homomorphism of $\mathscr{S}$ to a discrete measured groupoid $\left(\mathscr{Q}^{\prime}, \nu^{\prime}\right)$ with ker $\theta^{\prime} \supseteq \mathscr{R}$. Since $\theta$ is class-surjective, for each $\gamma \in \mathscr{Q}$ and each $x \in X$ with $\theta(x)=s(\gamma)$, we may choose $\phi(x) \in X$ with $\theta(\phi(x), x)=\gamma$; by [10], we may assume $\phi$ is Borel. Note $x \rightarrow \theta^{\prime}(\phi(x), x)$ is constant on $\mathscr{R}$-classes in $\alpha^{-1}(s(\gamma))$; but $\mathscr{R}$ is ergodic on $\left(\alpha^{-1}(y), \mu_{y}\right)$ for each $y \in Y$, so that $\theta^{\prime}(\phi(x), x)$ is equal $\mu_{s(\gamma)}$-a.e. to a constant which we denote by $\kappa(\gamma)$.

Clearly $\kappa$ is Borel and respects the measure classes in 2 and $2^{\prime}$. To see that it a homomorphism, let $\left(\gamma_{1}, \gamma_{2}\right) \in \mathscr{Q}^{(2)}$, and choose Borel maps $\phi_{1}, \phi_{2}$ with $\theta\left(\phi_{j}(x), x\right)=\gamma_{j}$ for $x \in \alpha^{-1}\left(s\left(\gamma_{j}\right)\right), j=1,2$. Since $s\left(\gamma_{1}\right)=r\left(\gamma_{2}\right), \phi_{2}(x) \in \alpha^{-1}\left(s\left(\gamma_{1}\right)\right)$ 
for $x \in \alpha^{-1}\left(s\left(\gamma_{2}\right)\right)$, so $\left(\phi_{1} \circ \phi_{2}(x), x\right) \in \mathscr{S}$ is defined for $x \in \alpha^{-1}\left(s\left(\gamma_{2}\right)\right)$. Since $\theta\left(\phi_{1} \circ \phi_{2}(x), x\right)=\gamma_{1} \gamma_{2}$ and

$$
\theta^{\prime}\left(\phi_{1} \circ \phi_{2}(x), x\right)=\theta^{\prime}\left(\phi_{1} \circ \phi_{2}(x), \phi_{2}(x)\right) \theta^{\prime}\left(\phi_{2}(x), x\right)
$$

we obtain $\kappa\left(\gamma_{1} \gamma_{2}\right)=\kappa\left(\gamma_{1}\right) \kappa\left(\gamma_{2}\right)$ as required.

We also include the following result, which is intended as further justification for the nomenclature 'normal subrelation'. Recall that if $\mathscr{S}$ is an ergodic equivalence relation on $(X, \mu)$, if $G$ is a real algebraic group and $\alpha \in Z^{1}(\mathscr{S}, G)$, then there is an algebraic subgroup $H$ of $G$ such that

(a) $\alpha$ is cohomologous to $\beta$, where $\beta(\mathscr{P}) \subseteq H$;

(b) $H$ is minimal among algebraic subgroups satisfying (a), and

(c) if $\alpha$ is cohomologous to $\gamma$ and $\gamma(\mathscr{S}) \subseteq L$ where $L \subseteq G$ is algebraic, then $L$ contains a conjugate of $H$.

Thus $H$ is unique up to conjugacy; it (or more precisely its conjugacy class) is called the algebraic hull of $\alpha$. (See [24] for further discussion.)

ThEOREM 2.6. Let $\mathscr{R} \subseteq \mathscr{S}$ be normal and ergodic, and let $\alpha \in Z^{1}(\mathscr{S}, G)$ be a cocycle, where $G$ is real and algebraic, and suppose that the algebraic hull of $\alpha$ is $G$. Then the algebraic hull $H$ of $\left.\alpha\right|_{\mathscr{R}}$ is normal in $G$.

Proof. We know $\mathscr{S}$ is generated by $\mathscr{R}$ and a countable family $\left\{\phi_{i}\right\} \subseteq$ Aut $_{\mathscr{S}}(\mathscr{R})$ from Theorem 2.2, and Lemma 1.3. Since for $(x, y) \in \mathscr{R}$ and $\phi \in$ Aut $_{\mathscr{S}}(\mathscr{R})$ we have

$$
\alpha(y, \phi y)=\alpha(y, x) \alpha(x, \phi x) \alpha(\phi x, \phi y)
$$

and $\alpha(\mathscr{R}) \subseteq H$, we conclude $\alpha(y, \phi y) \in H \alpha(x, \phi x) H$, so that if [ ]: $G \rightarrow H \backslash G / H$ is the canonical map, $x \in X \rightarrow[\alpha(x, \phi x)]$ is constant on $\mathscr{R}$-classes. Since $H \backslash G / H$ is countably separated $[24$, p. 34], we may assume $[\alpha(x, \phi x)]$ is constant, so there exists $g_{\phi} \in G$ and measurable maps $h_{\phi}, k_{\phi}: X \rightarrow H$ such that $\alpha(y, \phi y)=$ $h_{\phi}(x)^{-1} g_{\phi} k_{\phi}(x)$ for all $x$. From

$$
\alpha(x, \phi x)^{-1} \alpha(x, y) \alpha(y, \phi y)=\alpha(\phi x, \phi y),
$$

we obtain

$$
k_{\phi}(x)^{-1} g_{\phi}^{-1} h_{\phi}(x) \alpha(x, y) h_{\phi}(y)^{-1} g_{\phi} k_{\phi}(y)=\alpha(\phi x, \phi y)
$$

so that (recalling $\alpha(\mathscr{R}) \subseteq H$ and $k_{\phi}(X) \subseteq H$ ),

$$
g_{\phi}^{-1} h_{\phi}(x) \alpha(x, y) h_{\phi}(y)^{-1} g_{\phi} \in H \quad \text { for }(x, y) \in \mathscr{R} .
$$

Now since $H$ is the algebraic hull of $\left.\alpha\right|_{\mathscr{R}},\left\{h_{\phi}(x) \alpha(x, y) h_{\phi}(y)^{-1}:(x, y) \in \mathscr{R}\right\}$ generates $H$ as an algebraic group and we conclude that $g_{\phi}^{-1} H g_{\phi} \subseteq H$. But the algebraic hull of $\alpha$ is $G$, so $H \cup\left\{g_{\phi_{i}}\right\}$ generates $G$ as an algebraic group, and $H$ is thus normal.

The crucial property of algebraic groups used in the argument above is the fact that $H \backslash G / H$ is countably separated. The following assertion may be proved by the same technique as used above.

TheOREM 2.7. Suppose $\mathscr{R}$ is normal in $\mathscr{S}$ and ergodic, let $G$ be a locally compact group with $H$ a closed subgroup and let $\alpha \in Z^{1}(\mathscr{S}, G)$. Suppose that

(a) $\alpha$ is not equivalent to a cocycle with values in a proper closed subgroup of $G$, 
(b) $\left.\alpha\right|_{\mathscr{R}}$ cobounds into $H$, but not into any strictly smaller closed subgroup, and

(c) $H \backslash G / H$ is countable separated.

Then $H$ is normal in $G$.

We note that the hypotheses (a) and (b) of the Theorem are met if $\mathscr{S} \times{ }_{\alpha} G$ and $\mathscr{R} \times{ }_{\alpha} H$ are ergodic and hypothesis (c) is met if $G$ is discrete or compact. We also note that if $\alpha \in Z^{1}(\mathscr{S}, K)$ where $K$ is compact, then we can find closed subgroups $G, H$ of $K$ to which the theorem applies (see [25]).

We now turn to examples of normal subrelations and their quotients.

Example 2.8. Let $\mathscr{S}$ be given on $(X, \mu)$ as usual, and let $\mathscr{R} \subseteq \mathscr{S}$ be a smooth relation. As already noted, $\mathscr{R}$ is normal in $\mathscr{S}$. Let $F \subseteq X$ be a Borel set which meets almost all equivalence classes of $\mathscr{R}$ in a singleton; note $\mu(F)>0$, and set $2=\left.\mathscr{Y}\right|_{F}$, the reduction of $\mathscr{S}$ to $F$. For each $x \in X$, let $\theta(x) \in F$ be the unique point in $\mathscr{R}(x) \cap F$. Observe that $(x, y) \in \mathscr{S} \rightarrow(\theta(x), \theta(y)) \in \mathscr{Q}$ is a homomorphism with kernel $\mathscr{R}$, and which is class surjective; $\theta$ also has the universal property in Theorem 2.2(d), since if $\theta^{\prime}: \mathscr{S} \rightarrow \mathscr{Q}^{\prime}$ is a homomorphism with $\operatorname{ker} \theta^{\prime} \supseteq \mathscr{R}$, the restriction $\kappa$ of $\theta^{\prime}$ to 2 provides the factorization $\theta^{\prime}=\kappa \circ \theta$. Thus the quotient is $\left(\left.\left.\mathscr{P}\right|_{F^{\prime}} \mu\right|_{F}\right)$.

Example 2.7. If $H$ is a countable group acting freely and ergodically on $(X, \mu)$, generating an equivalence relation $\mathscr{S}$, and if $K \subseteq H$ is a normal subgroup which generates $\mathscr{R} \subseteq \mathscr{Y}$, then $\mathscr{R}$ is normal in $S$. Indeed if $\left\{h_{j}\right\}$ are a complete set of coset representatives for $K$ in $H$, so $H=\bigcup_{j} K h_{j}$, and $\phi_{j}(x)=h_{j} x$, then $\left\{\phi_{j}\right\}$ is a set of choice functions for $\mathscr{R} \subseteq \mathscr{S}$ (whether $K$ is normal or not), and $\phi_{j} \in \operatorname{Aut}_{\mathscr{S}}(\mathscr{R})$ whenever $K$ is normal. Note that in this case, with the notation used in the construction of the quotient groupoid $\mathscr{S} / \mathscr{R}, j *_{k} k=j k$, where $j k$ is the product in $H / K$. Note the parameter space $(Y, \nu)$ for the ergodic decomposition of $\mathscr{R}$ carries a natural non-singular action of $G=H / K$; the quotient groupoid is nothing but $G \times Y$, and the quotient map $\theta$ is given by $\theta(h x, x)=(h K, \alpha(x))$ where $\alpha$ is the canonical map from $X$ to $Y$. In view of the fact that the quotient $\mathscr{S} / \mathscr{R}$ depends only on the isomorphism class of the pair $\mathscr{R} \subseteq \mathscr{S}$, we obtain the following result.

COROLlaRY 2.10. For $j=1,2$, let $H_{j}$ be a countable group acting freely and nonsingularly on $\left(X_{j}, \mu_{j}\right)$, and let $K_{j} \subseteq H_{j}$ be normal subgroups which act ergodically. Suppose $\theta: X_{1} \rightarrow X_{2}$ is a Borel bijection with $\mu_{2} \circ \theta \sim \mu_{1}$ and

$$
\theta\left(H_{1} x\right)=H_{2} \theta(x), \quad \theta\left(K_{1}, x\right)=K_{2}(\theta x) \text { for } \mu \text { a.a. } x
$$

Then $H_{1} / K_{1}$ is isomorphic with $H_{2} / K_{2}$.

Example 2.11. Let $(2, \nu)$ be an arbitrary discrete measured ergodic groupoid. We will construct a pair $\mathscr{R} \subseteq \mathscr{S}$ of countable non-singular equivalence relations with $\mathscr{S}$ ergodic, $\mathscr{R}$ normal in $\mathscr{S}$ and $\mathscr{S} / \mathscr{R}$ isomorphic with $(\mathscr{Q}, \nu)$. For $y \in Y=\mathscr{Q}^{(0)}$ let $\mathscr{2}^{y}=\{\gamma \in \mathscr{Q}: r(\gamma)=y\}$, let $I(y)=\mathscr{2}^{y} \times N$, let $X(y)=\prod_{I(y)} Z_{2}$, with $\mu_{y}$ the Haar measure on $X(y)$, and let $\mathscr{R}_{y}$ be the equivalence relation on $X(y)$ defined by the natural action of the subgroup $\bigsqcup_{l(y)} Z_{2}$. For $\gamma \in \mathscr{Q}$ with $s(\gamma)=y, r(\gamma)=y^{\prime}$, define an isomorphism $t_{\gamma}: X(y) \rightarrow X\left(y^{\prime}\right)$ by $\left(t_{\gamma} x\right)\left(\gamma_{1}, n\right)=x\left(\gamma^{-1} \gamma_{1}, n\right)$; observe that $t_{\gamma}$ is an isomorphism of $\mathscr{R}_{y}$ with $\mathscr{R}_{y^{\prime}}$, that $t_{\gamma_{1} \gamma_{2}}=t_{\gamma_{1}} t_{\gamma_{2}}$ for $\left(\gamma_{1}, \gamma_{2}\right) \in \mathscr{Q}^{(2)}$, and that if $r(\gamma)=$ $s(\gamma)=y, t_{\gamma}$ is an outer automorphism of $\mathscr{R}_{y}$ unless $\gamma$ is a unit. By choosing (arbitrary) 
bijections of $I(y)$ with $N$, we obtain an isomorphism of $Y \times \prod_{N} Z_{2}$ with $X=$ $\bigcup_{y} X(y)$, and hence a standard Borel structure on $X$. We let $\mu=\int_{Y}^{\oplus} \mu_{y} d \nu(y)$ on $X$, and let $p: X \rightarrow Y$ be the obvious surjection. Define $\mathscr{R}$ on $X$ by $\left(x, x^{\prime}\right) \in \mathscr{R}$ if and only if $p(x)=p\left(x^{\prime}\right)$ and $\left(x, x^{\prime}\right) \in \mathscr{R}_{p(x)}$. Define $\mathscr{S}$ on $X$ by $\left(x, x^{\prime}\right) \in \mathscr{S}$ if and only if there is a $\gamma \in \mathscr{Q}$ with $p(x)=r(\gamma), p\left(x^{\prime}\right)=s(\gamma)$ and $\left(x, t_{\gamma}\left(x^{\prime}\right)\right) \in \mathscr{R}_{p(x)}$. It is routine to verify that $\mathscr{S}$ is ergodic on $(X, \mu)$. If we define $\theta: \mathscr{S} \rightarrow \mathscr{Q}$ by $\theta\left(x, x^{\prime}\right)=\gamma$ whenever $p(x)=r(\gamma), p\left(x^{\prime}\right)=s(\gamma)$ and $\left(x, t_{\gamma}\left(x^{\prime}\right)\right) \in R_{p(x)}$, it is straightforward to verify that $\theta$ is class-surjective and has kernel $\mathscr{R}$. It follows from the proof of Theorem 2.2 that $\mathscr{R}$ is normal in $\mathscr{S}$, and from the construction of $\mathscr{P} / \mathscr{R}$ in Theorem 2.2 that $\mathscr{S} / \mathscr{R}$ is indeed isomorphic with $(\mathscr{Q}, \nu)$. We remark that in this example, $\mathscr{R}$ is hyperfinite and of type $\mathrm{II}_{1}$, and the type of $\mathscr{P}$ is controlled in the obvious way by the type of the equivalence relation associated to $(2, \nu)$. Finally, we note that in an obvious sense $\mathscr{S}$ is the skew product of the family of relations $y \in \mathscr{2}^{(0)} \rightarrow \mathscr{R}_{y}$ by the 'action' of 2; however we will not pursue this here.

We now focus attention on normal ergodic subrelations. We first remark that if $\mathscr{R} \subseteq \mathscr{P}$ is normal and ergodic, the group $Q=\mathscr{P} / \mathscr{R}$ is (isomorphic to) the group associated in $[25,9]$ to the extension $p: \hat{\mathscr{R}} \rightarrow \mathscr{S}$-we leave the verification to the reader.

For our next result, we need the following observation. If $\mathscr{R}$ on $(X, \mu)$ is a discrete measured equivalence relation, $G$ a countable group and $g \rightarrow \phi_{g}$ a map from $G$ to the non-singular maps on $(X, \mu)$ with the properties.

(a) if $\left(x, x^{\prime}\right) \in \mathscr{R},\left(\phi_{\mathrm{g}}(x), \phi_{\mathrm{g}}\left(x^{\prime}\right)\right) \in \mathscr{R}$ for all $g \in G$, and

(b) $\left(\phi_{\mathrm{g}} \circ \phi_{\mathrm{h}}(x), \phi_{\mathrm{gh}}(x)\right) \in \mathscr{R}$ for all $x \in X$ we may define a new non-singular equivalence relation $\mathscr{S}$ on $(X, \mu)$ by $\left(x, x^{\prime}\right) \in \mathscr{S}$ if and only if, for some $g \in G$, $\left(x, \phi_{g}\left(x^{\prime}\right)\right) \in \mathscr{R}$. We refer to such a map $\phi$ as a near action of $G$ on $\mathscr{R}$ by endomorphisms, (or automorphisms if $\phi_{g} \in$ Aut $(\mathscr{R})$ for all $g \in G$ ), and as an outer near action if $\left(\phi_{g}(x), x\right) \notin \mathscr{R}$ for all $g \neq e$. We write $\mathscr{S}=\mathscr{R} \vee_{\phi} G$, and note that if the near action is outer, there is a homomorphism $\theta: \mathscr{R} \vee_{\phi} G \rightarrow G$ defined by $\theta\left(x, x^{\prime}\right)=g$ if $\left(x, \phi_{g}\left(x^{\prime}\right)\right) \in \mathscr{R}$; clearly $\theta$ is class surjective, has kernel $\mathscr{R}$, and $\left(\mathscr{R} \vee_{\phi} G\right) / \mathscr{R}$ is precisely $G$.

THEOREM 2.12. Let $\mathscr{R} \subseteq \mathscr{S}$ be normal and ergodic with quotient group $Q$. Then

(a) there is an outer near action $\phi$ by $Q$ as enolomorphism of $\mathscr{R}$ such that $\mathscr{S}=\mathscr{R} \vee_{\phi} Q$;

(b) the index cocycle $\sigma \in Z^{1}\left(S, \sum(Q)\right)$ may be realized as $\sigma=\rho \circ \theta$, where $\theta$ is the quotient homomorphism and $\rho$ is the Cayley representation of $Q$ in $\sum(Q)$ as right translations.

Proof. Since the quotient map $\theta$ is class-surjective, we may choose Borel maps $\phi_{q}: X \rightarrow X$ with $\left(\phi_{q}(x), x\right) \in \mathscr{S}$ and $\theta\left(\phi_{q} x, x\right)=q$. It is automatic that $q \rightarrow \phi_{q}$ provides an outer near action by (non-singular) endomorphisms, and that $\mathscr{S}=\mathscr{R} \vee_{\phi} Q$.

Further, since $\left\{\phi_{q}\right\}_{q \in Q}$ are choice functions for $\mathscr{R} \subseteq \mathscr{S}$, we may calculate the associated cocycle $\sigma$ by observing that $\sigma\left(\phi_{q} x, x\right)(p)=r$ if and only if $\mathscr{R}\left(\phi_{p}(x)\right)=$ $\mathscr{R}\left(\phi_{r} \phi_{q}(x)\right)$ and hence if and only if $p=r q$, so $\sigma\left(\phi_{q} x, x\right)$ is right translation by $q$ on $Q$ as required.

THEOREM 2.13. Let $\mathscr{R}$ be normal and ergodic in $\mathscr{S}$ with $\mathscr{S} / \mathscr{R}=Q$, and let $\left\{\phi_{q}\right\}$ be a near action of $Q$ on $\mathscr{R}$ with $\mathscr{R} \vee_{\phi} Q=\mathscr{S}$ as in Theorem 2.10. For each subgroup $H$ of 
$Q$, let $\mathscr{T}_{H}=\mathscr{R} \vee_{\phi} H$. Then $H \rightarrow \mathscr{T}_{H}$ is a lattice isomorphism from the subgroups of $Q$ the subrelations $\mathscr{T}$ of $S$ with $\mathscr{R} \subseteq \mathscr{T} \subseteq \mathscr{T}$, and $\mathscr{R}$ is normal in $\mathscr{T}_{H}$ with $\mathscr{T}_{H} / \mathscr{R}=\boldsymbol{H}$. Further, $\mathscr{T}_{H}$ is normal in $\mathscr{S}$ if and only if $H$ is normal in $Q$.

Proof. For each subgroup $H$ of $Q$, it is clear that $\mathscr{T}_{H}$ is an equivalence relation with $\mathscr{R}$ normal in $\mathscr{T}_{H}$ and $\mathscr{T}_{H} / \mathscr{R}=H$. Conversely suppose $\mathscr{R} \subseteq \mathscr{T} \subseteq \mathscr{Y}$, and let

$$
Q_{x}=\left\{q \in Q:\left(\phi_{q} x, x\right) \in \mathscr{T}\right\},
$$

for $x \in X$. Since $\phi_{q} \in \operatorname{End}_{\mathscr{f}}(\mathscr{R})$ for each $\phi$ we find $Q_{x}=Q_{y}$ for $(x, y) \in \mathscr{R}$, and by ergodicity of $\mathscr{R}$, we conclude there is a subset $H$ of $Q$ such that $Q_{x}=H$ a.e. Since $\mathscr{T}$ is an equivalence relation, it follows that $H$ is in fact a subgroup; trivially $\mathscr{T}=\mathscr{T}_{H}$ (except possibly on a null set). By construction, $H \rightarrow \mathscr{T}_{H}$ is a lattice isomorphism.

If $H$ is normal in $Q$, it is clear that $\mathscr{T}_{H}$ is normal in $\mathscr{S}$ (cf. Example 2.7). Conversely if $\mathscr{T}_{H}$ is normal in $\mathscr{S}$ and $\left\{q_{k}\right\}$ are coset representatives for $H$ in $Q$ (so $Q=\bigcup H q_{k}$ ), then if $\psi_{k}=\phi_{q_{k}},\left\{\psi_{k}\right\}$ are choice functions for $\mathscr{T}_{H} \subseteq \mathscr{T}$, and the associated cocycle $\sigma_{H}$ may be realized as $\sigma_{H}(x, y)=t(\theta(x, y))$, where $\theta: \mathscr{S} \rightarrow Q$ is the canonical homomorphism, and $t$ is the action of $Q$ on $H \backslash Q$ by right translation. If $\sigma_{H}$ cobounds on $\mathscr{T}_{H}, \sigma_{H}(x, y)=\nu_{x} \nu_{y}^{-1}$ for $\nu: X \rightarrow \sum(H \backslash Q)$; then, since ker $\theta=\mathscr{R}$ and $\mathscr{R}$ is ergodic, $\nu_{x}$ is constant a.e. We conclude $\sigma_{H}=$ identity, so that for all $k \in H \backslash Q$ and $h \in H, \mathscr{R}\left(\psi_{k}(h x)\right)=\mathscr{R}\left(\psi_{k}(x)\right)$; thus $H g_{k} h=H g_{k}$ for all $k$ and $h \in H$, from which normality of $H$ follows easily.

We will need in $\S 4$ a notion stronger than that of normality; we will (provisionally) call this strong normality.

Definition 2.14. Let $\mathscr{R} \subseteq \mathscr{S}$ be a subrelation. We say $\mathscr{R}$ is strongly normal in $\mathscr{S}$ if $\operatorname{Aut}_{\mathscr{S}}(\mathscr{R})=\operatorname{Int}(\mathscr{P})$.

Since always Int $(\mathscr{R}) \subseteq \operatorname{Aut}_{\mathscr{S}}(\mathscr{R}) \subseteq \operatorname{Int}(\mathscr{S})$, Aut $_{\mathscr{R}}(\mathscr{P})$ gives rise to an equivalence relation, say $U$, with $\mathscr{R} \subseteq U \subseteq \mathscr{Y}$. One easily sees that $U$ is generated by a countable subgroup of Int $(\mathscr{S})$, so $U$ is measurable. Strong normality is thus equivalent to equality of $\mathcal{U}$ and $\mathscr{S}$. Clearly strong normality implies normality, but the converse does not hold as the following example (which we owe to K. Schmidt) shows. In the example $\mathscr{S} / \mathscr{R}$ is a measured equivalence relation, and thus far from being a group.

THEOREM 2.15. Let $\mathscr{R} \subseteq \mathscr{S}$ be as usual on $(X, \mu)$, with $\mathscr{R}$ normal in $\mathscr{S}$, and suppose that $\mathscr{S} / \mathscr{R}$ is an equivalence relation $\mathscr{T}$ on $(Y, \nu)$. Then, the equivalence relation generated by Aut $_{\mathscr{S}}(\mathscr{R})$ on $X$ (called $\mathcal{U}$ above) is precisely $\left\{\left(x, x^{\prime}\right): J\left(x, x^{\prime}\right)=0\right\}$, where $J$ is the information cocycle of the pair $(\mathscr{R}, \mathscr{T})$ as in [20]. In particular, $\mathscr{R}$ is strongly normal in $\mathscr{S}$ if and only if $J \equiv 0$.

Proof. Let $\theta: \mathscr{S} \rightarrow \mathscr{T}$ be the quotient map. We regard $\theta$ as a map from $X$ to $Y$ with $\mu \circ \theta^{-1}=\nu, \theta(S(x))=\mathscr{T}(\theta(x))$ for all $x \in X$, and $\operatorname{ker} \theta=\mathscr{R}$. We let $\delta_{\mathscr{S}}, \delta_{\mathscr{T}}$ denote the Radon-Nikodym cocycles of $\mathscr{S}, \mathscr{T}$ with respect to the measures $\mu, \nu$ representively, and note $\delta_{\mathscr{S}} \equiv 1$, and $J=\log \delta_{\mathscr{T}} \circ \theta^{(2)}$.

Note that if $\phi \in \operatorname{End}_{\mathscr{P}}(\mathscr{R})$, then the map $x \in X \rightarrow \theta(\phi(x))$ is constant on $\mathscr{R}$-classes, so there is a unique map $\tilde{\phi}: Y \rightarrow Y$ which is non-singular for $\nu$ and such that $\tilde{\phi} \circ \theta=\theta \circ \phi$. a.e. Then we may assume any such endomorphism carries fibres of $\phi$ 
to fibres of $\phi$. We will also write $\mu=\int_{Y}^{\oplus} \mu_{y} d \nu(y)$ for the decomposition of $\mu$ with respect to the map $\theta$, and note for later reference that $\mu_{y}$ may be assumed ergodic for $\mathscr{R}$ for each $y \in Y$, and that $\left(\mathscr{R}, \mu_{y}\right)$ is of uniformly type $\mathrm{II}_{1}$ or $\mathrm{I}_{n}$, for some $n$.

Suppose now $\phi \in \operatorname{Aut}_{\varphi}(\mathscr{R})$; we show that $J(\phi x, x)=0$ a.e. Observe that for almost all $y, \mu_{\tilde{\phi} y} \circ \phi$ is an invariant probability measure for $\mathscr{R}$ on $\phi^{-1}(y)$, and that $\mu_{\tilde{\phi} y} \circ \phi$ is equivalent to $\mu_{y}$ by the essential uniqueness of the decomposition of $\mu$ with respect to $\phi$. Thus $\mu_{\tilde{\phi} y} \circ \phi=\mu_{y}$ a.e. In view of the relation

$$
\delta_{\mathscr{S}}(\phi x, x)=\frac{d \mu_{\theta(\phi x)} \circ \phi}{d \mu_{\phi(x)}}(x) \delta_{\mathscr{T}}(\theta(\phi x), \theta(x))
$$

we conclude $\delta_{\mathscr{g}}(\theta(\phi x), \theta(x))=1$ a.e. since $\delta_{\mathscr{S}}=1$ and $\mu_{\tilde{\phi} y} \circ \phi=\mu_{y} \nu$-a.e. Thus $(\phi x, x) \in \operatorname{ker} J$.

Conversely, observe that $\mathscr{R} \subseteq$ ker $J$ is normal so that, by a slight modification of the arguments of 1.1 (to allow for the possible non-ergodicity of ker $J$ ) we may assume there exists endomorphisms $\left\{\psi_{j}\right\}_{j} \subseteq \operatorname{End}_{\mathscr{S}}(\mathscr{R})$ with $\left(x^{\prime}, x\right) \in \operatorname{ker} J$ if and only if $\left(x^{\prime}, \psi_{j}(x)\right) \in \mathscr{R}$ for some $j$. It thus suffices to show that if $\psi \in \operatorname{End}_{\mathscr{S}}(\mathscr{R})$ is such that $(\psi(s), x) \in \operatorname{ker} J$ for all $x \in X$, then there exists $\phi \in \operatorname{Aut}_{\mathscr{P}}(\mathscr{R})$ such that $(\phi(x), \psi(x)) \in \mathscr{R}$ for almost all $x$.

To see this, observe that since $(\psi x, x) \in \mathscr{S}$ for all $x$, there is a partition $\left\{E_{k}\right\}$ of $X$ such that $\psi_{k}=\left.\psi\right|_{E_{k}}$ is 1-1 for each $k$ (see [10]). Since $\psi_{k}$ preserves $\left.\mu\right|_{E_{k}}$, and since $\delta_{\mathscr{T}}(\tilde{\psi} y, y)=1$ a.e., we conclude that $\mu_{\tilde{\psi} k} \circ \psi_{k}\left(E_{k}\right)=\mu_{y}\left(E_{k}\right)$ a.e. in $y$ for all $k$, so that $\sum_{k} \mu_{\tilde{\psi} y}\left(\psi_{k}\left(R_{k}\right)\right)=1$ a.e. in $y$. Using the ergodicity of $\left(\mathscr{R}, \mu_{\tilde{\psi} y}\right)$ and the fact that it is finite, we conclude there exists partitions $\left\{F_{k}^{y}\right\}$ of $\theta^{-1}(\tilde{\psi}(y))$ and elements $V_{k}^{y} \in$ $[R]_{\left.\mu_{\psi(x)}\right)}$ such that $V_{k}^{y}\left(\psi_{k}\left(E_{k} \cap \phi^{-1}(y)\right)=F_{k}^{y}\right.$. The map $\phi: X \rightarrow X$ defined by $\phi(x)=$ $V_{k}^{y} \psi_{k}(x)$ for $x \in E_{k} \cap \phi^{-1}(y)$ is thus a bijection; and routine arguments show we may choose the sets $\left\{F_{k}^{y}\right\}$ and maps $V_{k}^{y}$ in such a manner that $\phi$ and $\phi^{-1}$ are measurable. Since $(\phi(x), \psi(x)) \in \mathscr{R}$ for each $x \in X$, by construction, the proof is complete.

We note that [20] provides a variety of examples of quotient relations $\mathscr{T}$ for which the information cocycle $J$ is not trivial. Also, Example 2.11 shows how to construct a pair $\mathscr{R} \subseteq \mathscr{S}$ for any given $\mathscr{T}$.

Again, we close the section with comments regarding the situation where $\mathscr{S}$ is not of type $\mathrm{II}_{1}$. All the material up to and including Theorem 2.11 holds, as stated and as proved, in the general context. If $\mathscr{S}$ is of type $\mathrm{II}_{\infty}$ (or indeed if Lemma 1.3 holds for $\mathscr{R} \subseteq \mathscr{S}$ ), we may strengthen the conclusion of Theorem $2.2(\mathrm{~b})$ to read 'There are choice functions $\left\{\phi_{j}\right\}$ for $\mathscr{R} \subseteq \mathscr{S}$ with $\phi_{j} \in \mathrm{Aut}_{\mathscr{S}}(\mathscr{R})$ for all $j$ '. Clearly, Theorem 2.13 is special to the case where $\mathscr{S}$ is of type $\mathrm{II}_{1}$.

\section{Classification results}

Throughout this section, $\mathscr{S}$ denotes as usual, a discrete ergodic equivalence relation on $(X, \mu)$, which is of type $\mathrm{II}_{1}$. We refer to [3] for a discussion of amenability of such relations.

Theorem 3.1. Suppose $\mathscr{S}$ is amenable. Then for each $N<\infty$ there is a bijective 
correspondence between the ergodic subrelations $\mathscr{T}$ of $\mathscr{S}$ of index $N$ (up to automorphisms of $\mathscr{S}$ ), and the conjugacy classes of transitive subgroups of $\sum(N)$.

Proof. Let $\mathscr{R} \subseteq \mathscr{S}$ be ergodic of index $N$, and let a $\sigma \in Z^{1}(\mathscr{S}, \Sigma(N))$ be an index cocycle for $\mathscr{R} \subseteq \mathscr{S}$. As noted in [25], there is a unique (up to conjugacy) subgroup $K$ of $\sum(N)$ such that $\sigma$ cobounds into $K$ and, as a cocycle into $K$ is ergodic. The ergodicity of $\mathscr{R}$ and hence $\mathscr{R} \times_{\sigma} J$ implies that $K$ acts transitively on $J=$ $\{0,1, \ldots, N-1\}$.

Conversely, if $K$ is a transitive subgroup of $\sum(N)$, then by [12], there is a cocycle $\sigma \in Z^{1}(\mathscr{S}, K)$ which is ergodic, and this cocycle is unique up to cohomology and automorphisms of $\mathscr{S}$. Since $\sigma$ is ergodic into $K$ and $K$ acts transitively on $\{1,2, \ldots, N\}, \mathscr{S} \times_{\alpha} J$ is ergodic. By Theorem 1.6(a) there is thus an ergodic subrelation $\mathscr{R} \subseteq \mathscr{S}$ whose index cocycle is $\sigma$, and by Theorem 1.6(b), $\mathscr{R}$ is uniquely determined up to automorphisms of $\mathscr{S}$.

We note that in the Theorem above, the normal subrelations $\mathscr{R}$ of finite index $N$ in $\mathscr{S}$ correspond to transitive freely acting subgroups of $\sum(N)$ of minimal order. Theorem 3.1 has also been obtained (in the context of extensions) in [11].

THEOREM 3.2. Let 9 be amenable. Then for each countable amenable group $Q$, there is a normal ergodic subrelation $\mathscr{R}$ of $\mathscr{S}$ such that $\mathscr{S} / \mathscr{R}$ is isomorphic with $Q$; further, $\mathscr{R}$ is unique up to automorphisms of $\mathscr{S}$.

Proof. Suppose $Q$ is a given countable amenable group. By [12], there is an essentially unique ergodic $\sigma \in Z^{1}(\mathscr{S}, Q)$ and, again using Theorem 1.6, this determines an ergodic subrelation $\mathscr{R}$ of $\mathscr{S}$, unique up to automorphisms, with $\mathscr{S} / \mathscr{R}$ isomorphic to $Q$.

Conversely, suppose $\mathscr{R} \subseteq \mathscr{S}$ is a given normal ergodic subrelation with $\mathscr{S} / \mathscr{R}=Q$. In order to prove that $\mathscr{R}$ is uniquely determined up to automorphisms of $\mathscr{S}$ by $Q$, it suffices to show that $Q$ is amenable, and to then appeal to [12] and Theorem 1.6.

Let $\left\{p_{x}\right\}$ be a measurable, translation-invariant family of means on $\mathscr{P}$, as in [3]. For each $f \in \ell^{\infty}(Q)$ define $F$ on $\mathscr{S}$ by $F=f \circ \theta$, where $\theta$ is the quotient map of Theorem 2.2. Evidently $F$ is a Borel function. For each $x \in X$, let $\theta^{x}$ be the restriction of $\theta$ to $r_{\mathscr{S}}^{-1}(x)$ and note that $x \rightarrow p_{x}\left(f \circ \theta^{x}\right)$ is invariant under $\mathscr{R}$ since for $(x, y) \in \mathscr{R}$, $f \circ \theta^{x}=\lambda(x, y)\left(f \circ \theta^{y}\right)$ and since, for $(x, y) \in \mathscr{S}, p_{x} \circ \lambda(x, y)=p_{y}$ - here $\lambda(x, y)$ is the map from $\ell^{\infty}\left(r_{\mathscr{S}}^{-1}(y)\right)$ to $\ell^{\infty}\left(r_{\mathscr{S}}^{-1}(x)\right)$ defined by $(\lambda(x, y) \phi)(z)=\phi(z)$. Since $\mathscr{R}$ is ergodic, $p_{x}\left(f \circ \theta^{2}\right)$ is thus constant a.e.; we denote this constant by $m(f)$.

Evidently $m$ is a positive linear functional on $\ell^{\infty}(Q)$ with $m(1)=1$. Further, if $q \in Q$, one easily checks that $\lambda(y, x)\left(f \circ \theta^{x}\right)=\left({ }^{q} f\right) \circ \theta^{y}$ where $\theta(y, x)=q$ and ${ }^{q} f(p)=$ $f\left(q^{-1} p\right)$. We thus obtain $p_{x}\left(f \circ \theta^{x}\right)=p_{y}\left({ }^{a} f\right)$ whenever $\theta(y, x)=q$, so that $m(f)=$ $m\left({ }^{q} f\right)$ for each $q \in Q$ and $f \in \ell^{\infty}(Q)$. Thus $Q$ is amenable, as required.

We note that one may introduce a notion of 'relative amenability' for equivalence relations $\mathscr{R} \subseteq \mathscr{S}$ which corresponds under the duality operation $\mathscr{R} \rightarrow \hat{\mathscr{R}}$ of $\S 1$ to Zimmers notion of a relatively amenable extension. This, together with a number of other closely related considerations, will be taken up in a subsequent paper, [21]. 
We note one further consequence of our classification result.

COROllary 3.3. Let $\mathscr{S}$ be amenable, and let $\mathscr{R} \subseteq \mathscr{S}$ be normal and ergodic with $\mathscr{S} / \mathscr{R}=Q$. Then there is a homomorphism $\theta: Q \rightarrow$ Aut $(\mathscr{R})$ with $\mathscr{S}=\mathscr{R} \vee{ }_{\theta} Q$.

Proof. It suffices to produce, for any amenable discrete group $Q$, an outer action of $Q$ on a type $\mathrm{II}_{1}$ ergodic amenable relation $\mathscr{R}$ such that $\mathscr{S}=\mathscr{R} \vee{ }_{\theta} Q$ is of type $\mathrm{II}_{1}$. This is routine.

Perhaps surprisingly the requirement that $\mathscr{R}$ be amenable in Corollary 3.3 can be dropped.

THEOREM 3.4. Let $\mathscr{R}$ be a discrete measured ergodic equivalence relation of type $\mathrm{II}_{1}$, and suppose that $\phi: Q \rightarrow$ End $(\mathscr{R})$ is an outer near action of a countable amenable group $Q$ as endomorphisms of $\mathscr{R}$. Then there is an outer action $\psi: Q \rightarrow$ Aut $(\mathscr{R})$ with $\left(\phi_{q}(x), \psi_{q}(x)\right) \in \mathscr{R}$ for almost all $x \in X$.

The proof of this result will occupy the remainder of the section. Note that by elementary considerations (cf Lemma 1.3 ) we may assume that $\phi_{q} \in$ Aut ( $\mathscr{R}$ ) for all $q \in Q$. The strategy of the proof will be to produce inductively a sequence of maps $\phi^{n}: Q \rightarrow$ Aut $(\mathscr{R})$ such that

(i) for each $x \in X$ and $q \in Q, \phi_{q}^{n}(x)$ stabilizes as $n \rightarrow \infty$;

(ii) $\left(\phi_{q}^{n}(x), \phi_{q}(x)\right) \in \mathscr{R}$ for all $x$ and $n$;

(iii) for each $p, q \in Q, \phi_{p}^{n} \circ \phi_{p}^{n}=\phi_{p q}^{p}$ on sets $X_{p, q}^{n}$, with $\bigcup_{n} X_{p, q}^{n}=X(\bmod \mu)$.

If we can achieve this, and define $\psi_{q}(x)=\phi_{q}^{n}(x)$ for large enough $n, \psi$ will satisfy the conclusions of the Theorem.

To achieve the situation described above, we will use a 'cutting and stacking' technique, a standard construction in ergodic theory (see for example [18]). There are two complications. First in building the stack, we will not be able to use arbitrary maps between levels of the stack, but use maps whose graphs lie in $\mathscr{R}$-this will critically use ergodicity of $\mathscr{R}$. Secondly, the various levels of the stack will be labelled by elements of $Q$ rather than integers, and to achieve the desired conclusions we will need to use a variation of the ' $\varepsilon$-quasi-tiling' technique in amenable groups, as described in [18]. For the readers convenience, we recall some of the basic concepts and results from [18]. Throughout, $Q$ is a countable group; $|A|$ is the cardinality of a set $A$.

Definition 3.7. Finite subsets $A_{1}, \ldots, A_{n}$ of $Q$ are $\varepsilon$-disjoint (where $\varepsilon>0$ ) if there exists subsets $B_{j} \subseteq A_{j}, 1 \leq j \leq n$ which are disjoint and $\left|B_{j}\right| /\left|A_{j}\right|>1-\varepsilon, 1 \leq j \leq n$.

Definition 3.8. The finite subsets $A_{1}, \ldots, A_{n}$ of $Q$ are said to be $\varepsilon$-tiles if for each finite subset $F \subseteq Q$, there are finite subsets $C_{1}, \ldots, C_{n}$ of $Q$ such that

(i) for each $j$, the sets $\left\{\boldsymbol{A}_{j} c, c \in C_{j}\right\}$ are $\varepsilon$-disjoint;

(ii) the sets $A_{j} C_{j}, 1 \leq j \leq n$ are disjoint;

(iii) $\left|\left(\bigcup_{j=1}^{n} A_{j} C_{j}\right) \cap F\right|>(1-\varepsilon)|F|$.

By $\left[18, \S 1.3\right.$, Theorem 3], for any $\varepsilon>0$, there exists $n \in M$ and $\delta_{1}, \ldots, \delta_{n}>0$ such that whenever $A_{1} \subseteq A_{2} \subseteq \cdots \subseteq A_{n}$ are finite subsets of $Q$ with $e \in A_{1}$ and 
$A_{j+1}\left(A_{j} A_{j}^{-1}, \delta_{j}\right)$-invariant for $j=1,2, \ldots, n-1$, then $A_{1}, \ldots, A_{n}$ are $\varepsilon$-tiles (Here $B$ being $(D, \delta)$ invariant means that $\mid\{p \in B: D p \cap B \neq \varnothing$ and $D p \cap(Q-B) \neq \varnothing\} \mid<$ $\delta|B|$.

Note that if the set $F$ above is sufficiently invariant under $A_{1}, \ldots, A_{n}$ we can ensure (iv) $\bigcup_{j=1}^{n} A_{j} C_{j} \subseteq F$;

to do this, one simply replaces $C_{i}$ by $C_{i}^{\prime}=\left\{c \in C_{i}: A_{i} c \subseteq F\right\}$ and notes that (i), (ii), (iii) continue to hold.

The choice of $C_{1}, \ldots, C_{n}$ satisfying (i)-(iv) above is called an $\varepsilon$-tiling of $F$ by $A_{1}, \ldots, A_{n}$, and the members of $C_{1}, \ldots, C_{n}$ are called the $\varepsilon$-tiling centres. We shall want an additional property for our $\varepsilon$-tilings.

Definition 3.9. The $\varepsilon$-tiles $A_{1}, \ldots, A_{n}$ are said to have distribution $p=\left(p_{1}, \ldots, p_{n}\right)$, where $p_{j}>0, \sum_{j=1}^{n} p_{j}=1$ if for each $\delta>0$ there exists an $\eta$ such that for each $\left(\bigcup_{j=1}^{n} A_{j}, \eta\right)$-invariant finite set $F$, there is an $\varepsilon$-tiling $C_{1}, \ldots, C_{n}$ of $F$ such that (i)-(iv) holds, and

(v) for each $i,|| A_{i} C_{i}\left|/ \bigcup_{j=1}^{n} A_{j} C_{j}\right|-p_{i} \mid<\delta$.

LeMmA 3.10. Given $\varepsilon>0$, there exists $n \in N$ and $\delta_{1}, \ldots, \delta_{n}>0$ such that if $e \in A_{1} \subseteq$ $\cdots \subseteq A_{n}$ are finite sets with $A_{j+1}\left(A_{j} A_{j}^{-1}, \delta_{j}\right)$ invariant $(1 \leq j \leq n-1)$, then $A_{1}, \ldots, A_{n}$ are $\varepsilon$-tiles with some distribution $p=\left(p_{1}, \ldots, p_{n}\right)$.

Proof. Lemma 2 of $[18, \S 1.3]$ guarantees that for any sufficiently invariant set $F$, we may find a set $D_{m}$ with $\left\{A_{m} d: d \in D_{m}\right\} \varepsilon$-disjoint, contained in $F$, and $\left|\bigcup_{d \in D_{m}} A_{m} d\right| \geq$ $\varepsilon / 2|F|$ i.e. we can cover at least a fixed fraction of any sufficiently invariant set by almost disjoint translates of any of $A_{1}, \ldots, A_{m}$. We thus take $p_{n}=\varepsilon / 2, p_{j}=$ $\varepsilon / 2\left(1-\sum_{\ell=j+1}^{n} p_{\ell}\right)$ for $j \geq 2$, and $p_{1}=1-\sum_{\ell \geq 2} p_{\ell}$, and note that that byoosing $\varepsilon$-tiling centres $C_{n}, C_{n-1}, \ldots, C_{1}$ successively so that $A_{j} C_{j}$ covers a fraction within $\delta$ of $\varepsilon / 2$ of $F-\bigcup_{\ell>j} A_{\ell} C_{\ell}$, the proof of $[18, \S 1.2$, Theorem 6] still applies. We leave the details to the reader.

LEMMA 3.11. Let $Q$ be a countable amenable group. Then there are sequences $\varepsilon_{j} \rightarrow 0$, $n_{i} \in N$ such that for each $i$ there is a sequence $e \in A_{1}^{i} \subseteq A_{2}^{i} \subseteq \cdots \subseteq A_{n_{i}}^{i}$ of finite subsets of $Q$ and finite subsets $C_{j, k}^{i}$ of $Q\left(1 \leq j \leq n_{i}, 1 \leq k \leq n_{i+1}\right)$ and probability distributions $p^{i}=\left(p_{1}^{i}, \ldots, p_{n_{i}}^{i}\right)$ such that

(i) $A_{n_{i}}^{i} \subseteq A_{1}^{i+1}$, and each $A_{k}^{i+1}$ is $\left(A_{n_{i}}^{i}\left(A_{n_{i}}^{i}\right)^{-1}, \varepsilon_{i+1}\right)$-invariant,

(ii) the sets $\left\{A_{j}^{i} c: c \in C_{j, k}^{i}\right\}$ are $\varepsilon_{i}$-disjoint for each fixed $i$ and $k$;

(iii) the sets $\left\{A_{j}^{i} C_{j, k}^{i}: 1 \leq j \leq n\right\}$ are, for each $i$ and $k$, disjoint;

(iv) $\left|\bigcup_{j=1}^{n_{i}} A_{j}^{i} C_{j, k}^{i}\right|>\left(1-\varepsilon_{i}\right)\left|A_{k}^{i+1}\right|$ for each $i, k$;

(v) $\bigcup_{j=1}^{n_{i}} A_{j}^{i} C_{j, k}^{i} \subseteq A_{k}^{i+1}$ for each $i, k$;

(vi) ||$A_{j}^{i} C_{j, k}^{i}|/| \bigcup_{j=1}^{n_{i}} A_{j}^{i} C_{j, k}^{i}\left|-p_{j}^{i}\right|<\varepsilon_{i+1}$;

(vii) $\left|A_{j}^{i}\right|\left|C_{j, k}^{i}\right| /\left|A_{k}^{i+1}\right|<p_{j}^{i}$ for all $i, j, k$;

(viii) $\bigcup_{i=1}^{\infty} A_{n_{i}}^{i}=Q$.

Proof. We first show the existence of appropriate sequences satisfying (i)-(vi) and (viii). Let $\varepsilon_{1}>0$ be arbitrary and choose a system of $\varepsilon_{1}$-tiles $A_{1}^{1}, \ldots, A_{n_{1}}^{1}$, with some distribution $p^{1}$. Now take $\varepsilon_{2}>0$ and choose $\varepsilon_{2}$-tiles $A_{1}^{2}, \ldots, A_{n_{2}}^{2}$ with some distribu- 
tion $p^{2}$, and each $A_{k}^{2}$ sufficiently invariant under $\bigcup_{j=1}^{n_{1}} A_{j}^{1}$ so that by Lemma 3.10 there is an $\varepsilon_{1}$-tiling $C_{1, k}^{2}, \ldots, C_{n_{1}, k}^{2}$ of $A_{k}^{2}$ such that (iii)-(vi) are satisfied for $i=1$. Now proceed inductively; by amenability of $Q$ one can simultaneously ensure (viii).

In order to achieve (vii), note we may choose $\varepsilon_{i+1}<\varepsilon_{i} / 4 n_{i}$ for each $i$; using (iv) and (vi), we may choose each $A_{k}^{i+1}$ so that $1 /\left|C_{j, k}^{i}\right|<\varepsilon_{i+1}$. Now observe that if initially (iv) had been sharpened to $\| \bigcup_{j=1}^{n_{i}} A_{j}^{i} C_{j, k}^{i}\left|>\left(1-\varepsilon_{i} / 2\right)\right| A_{k}^{i+1} \mid$ (which we may clearly do) then by deleting one or more members of $C_{j, k}^{i}, k=1, \ldots, n_{i+1}$, we may bring each ratio $\left|A_{j}^{i}\right|\left|C_{j, k}^{i}\right| /\left|A_{k}^{i+1}\right|$ below $p_{j}^{i}$ without reducing $\| \bigcup_{j=1}^{n_{i}} A_{j}^{i} C_{j, k}^{i}|/| A_{k}^{i+1} \mid$ by more than $\varepsilon_{i} / 2$.

We now return to the proof of Theorem 3.4. For brevity, we use the term $\mathscr{R}$-map to mean a Borel map $\theta$ defined and bijective on a set $E \subseteq X$ with $(x, \theta(x)) \in \mathscr{R}$ for all $x \in E$. Recall that if $E, F \in X$ with $\mu(E)=\mu(F)$, there exists an $\mathscr{R}$-map $\theta$ with $\theta(E)=F(\bmod \mu)($ see for example [5]). We now establish the initial step in our inductive construction; we will keep the notation of Lemma 3.11.

LEMMA 3.12. Let $\phi=\phi^{0}$ be an outer near action of $Q$ as automorphisms of $(\mathscr{R}, \mu)$. Then there are disjoint sets $S_{j}^{1}, 1 \leq j \leq n_{1}$ of measure $p_{j}^{1} /\left|A_{j}^{1}\right|$ and an outer near action $\phi^{1}$ of $Q$ on $(\mathscr{R}, \mu)$ such that

(i) $\left(\phi_{q}^{0}(x), \phi_{q}^{1}(x)\right) \in \mathscr{R}$ for all $x \in X$ and $q \in Q$;

(ii) the map $(q, x) \in A_{j}^{1} \times S_{j}^{1} \rightarrow \phi_{q}^{1}(x)$ is bijective;

(iii) the sets $T_{j}^{1}=\bigcup_{q \in A_{j}^{1}} \phi_{q}^{1}\left(S_{j}^{1}\right)$ are disjoint, $1 \leq j \leq n_{1}$;

(iv) if $p, q$ and $p q \in A_{j}^{1}$, then $\phi_{p}^{1} \circ \phi_{q}^{1}=\phi_{p q}^{1}$ on $S_{j}^{1}$.

Proof. Choose arbitrary disjoint sets $S_{j}^{1}, 1 \leq j \leq n_{1}$ with $\mu\left(S_{j}^{1}\right)=p_{j}^{1} /\left|A_{j}^{1}\right|$. Choose $\mathscr{R}$-maps $\theta_{j}^{1}(p), p \in A_{j}^{1}$ such that $\left\{\theta_{j}^{1}(p) \phi_{p}^{0}\left(S_{j}^{1}\right), p \in A_{j}^{1}, 1 \leq j \leq n_{1}\right\}$ are disjoint-this is possible since we have $\sum_{j=1}^{n_{1}}\left|A_{j}^{1}\right| \mu\left(S_{j}^{1}\right)=\sum_{j=1}^{1} p_{j}^{1}=1$. Put $\beta_{j}(p)=\theta_{j}^{1}(p) \phi_{p}^{0}\left(p \in A_{j}^{1}\right)$, and $T_{j}^{1}=\bigcup_{p \in A_{j}^{l}} \beta_{j}(p) S_{j}^{1}$.

We now construct $\phi^{1}$. For $p \in A_{j}^{1}$ and $q \in A_{j}^{1}$ with $p q \in A_{j}^{1}$, and for $x \in S_{j}^{1}$, define $\phi_{p}^{1} \beta_{j}(q) x=\beta_{j}(p q) x$. For abitrary $p \in Q$, let $R_{j}^{1}(p)$ be those points of $T_{j}^{1}$ at which $\phi_{p}^{1}$ is now defined, so $R_{j}^{1}(p)=\varnothing$ if $p \notin A_{j}^{1}$, and

$$
R_{j}^{1}(p)=\bigcup_{q}\left\{\beta_{j}(q) S_{j}^{1}: q \in A_{j}^{1}, p q \in A_{j}^{1}\right) \quad \text { for } p \in A_{j}^{i} .
$$

Observe that in both cases, $\phi_{p}^{0}\left(T_{j}^{1}-R_{j}^{1}(p)\right)$ and $T_{j}^{1}-\phi_{p}^{1}\left(R_{j}^{1}(p)\right)$ have the same measure, so we may choose an $\mathscr{R}$-map $\gamma_{j}^{1}(p)$ sending the first of these sets bijectively to the second, and define $\phi_{p}^{1}=\gamma_{j}^{1}(p) \circ \phi_{p}^{0}$ on $T_{j}^{1}-R_{j}^{1}(p)$. By construction each $\phi_{p}^{1}$ is now an automorphism which leaves each $T_{j}^{j}$ invariant, and such that $\left(\phi_{p}^{1}(x), \phi_{p}^{0}(x)\right) \in \mathscr{R}$ for all $x$ and $p$, so that (i), (ii), (iii) of the Lemma are satisfied.

It remains to check (v). If $p, q$ and $p q \in A_{j}^{1}$, and $x \in S_{j}^{1}$, then

$$
\phi_{p}^{1} \circ \phi_{q}^{1}(x)=\phi_{p}^{1} \beta_{1}(q) x=\beta_{1}(p q) x=\phi_{p q}^{1}(x) \text {, }
$$

as required.

We now proceed to the inductive step.

Lemma 3.13. Assume that for $\ell \leq i$ we have disjoint sets $S_{j}^{\ell}, 1 \leq j \leq n_{\ell}$ of measure $p_{j}^{\ell} /\left|A_{j}^{\ell}\right|$ and outer near actions $\phi^{\ell}$ of $Q$ as automorphisms of $\mathscr{R}$ such that 
(i) $\phi^{\ell}$ is bijective from $A_{j}^{\ell} \times S_{j}^{\ell}$ to $T_{j}^{\ell}=\phi^{\ell}\left(A_{j}^{\ell}\right) S_{j}^{\ell}$;

(ii) the sets $T_{j}^{\ell}, 1 \leq j \leq n_{\ell}$ are disjoint;

(iii) $\phi_{p q}^{\ell}(x)=\phi_{p}^{\ell} \phi_{q}^{\ell}(x)$ for $x \in S_{j}^{\ell}$ provided $p, q$ and $p q \in A_{j}^{\ell}$.

Then we may choose disjoint subsets $S_{k}^{i+1}, 1 \leq k \leq n_{i+1}$ of measure $p_{k}^{i+1} /\left|A_{k}^{i+1}\right|$ and an outer near action $\phi^{i+1}$ of $Q$ as automorphisms of $\mathscr{R}$ such that conditions (i), (ii), (iii) above are satisfied (for $i+1$ in place of $i$ ) and

(iv) $\left(\phi_{p}^{i+1}(x), \phi_{p}^{i}(x)\right) \in \mathscr{R}$ for all $x \in X$;

(v) for $p \in A_{j}^{i-1}$ for some $j, \phi_{p}^{i}=\phi_{p}^{i+1}$ on a set of measure at least $\left(1-2 \varepsilon_{i}\right)\left(1-\varepsilon_{i}\right)$.

Proof. Choose arbitrary disjoint subsets $S_{k}^{i+1}, 1 \leq k \leq n_{i+1}$ of $X$ of measure $p_{k}^{i+1} /\left|A_{k}^{i+1}\right|$, and for $c \in C_{j, k}^{i}$, choose $\mathscr{R}$-maps $\theta_{j, k}^{i}(c)$ from $\phi_{c}^{i}\left(S_{k}^{i+1}\right)$ into $S_{j}^{i}$, so that the sets $\theta_{j, k}^{i} \phi_{c}^{i}\left(S_{k}^{i+1}\right)$ are disjoint for $c \in C_{j, k}^{i}$-this is possible since

$$
\begin{aligned}
\left|C_{j, k}^{i}\right| \mu\left(S_{k}^{i+1}\right) & =\left|C_{j, k}^{i}\right| p_{k}^{i+1} /\left|A_{k}^{i+1}\right| \\
& =\left(\left|A_{j}^{i}\right|\left|C_{j, k}^{i}\right| p_{k}^{i+1}\right) /\left(\left|A_{j}^{i+1}\right|\left|A_{j}^{i}\right|\right)<p_{k}^{i+1} p_{j}^{i} /\left|A_{j}^{i}\right|=p_{k}^{i+1} \mu\left|S_{j}^{i}\right|,
\end{aligned}
$$

where we have used Lemma 3.11(vii) to obtain the inequality.

For $c \in C_{j, k}^{i}$, choose subsets $B_{j, k}^{i}(c) \subseteq A_{j}^{i}$ such that the sets $\left\{B_{j, k}^{i}(c) c: c \in C_{j, k}^{i}\right\}$ are disjoint and $\left|B_{j, k}^{i}(c)\right| /\left|A_{j}^{i}\right|>\left(1-\varepsilon_{i}\right)$-the existence of such sets is guaranteed by Lemma 3.11(ii). Define a map $\beta_{i+1}$ on

$$
\left(\bigcup_{k=1}^{n_{i+1}}\left(\bigcup_{j=1}^{n_{i}} \bigcup_{c \in C_{j, k}^{i}} B_{j, k}^{i}(c) c\right) \times S_{k}^{i+1}\right)
$$

by

$$
\beta_{i+1}(p c, x)=\phi_{p}^{i} \theta_{j, k}^{i}(c) \phi_{c}^{i}(x) \text { for } p \in B_{j, k}^{i}(c), x \in S_{k}^{i+1} ;
$$

by the choice of $\theta_{j, k}^{i}$ and hypothesis (ii) of this Lemma, $\beta_{i+1}$ is injective. We extend $\beta_{i+1}$ to

$$
\bigcup_{k=1}^{n_{i+1}}\left(A_{k}^{i+1} \times S_{k}^{i+1}\right)
$$

by defining

$$
\beta_{i+1}(p, x)=\nu_{k}^{i+1}(p) \circ \phi_{p}^{i}(x) \quad \text { for } p \in A_{k}^{i+1}-\bigcup_{j=1}^{n_{i}} \bigcup_{c \in C_{j, k}^{i}} B_{j, k}^{i}(c) c \text { and } x \in S_{k}^{i+1} \text {, }
$$

where $\nu_{k}^{i+1}(p)$ is an $\mathscr{R}$-map chosen so that the images $\beta_{i+1}\left(p, S_{k}^{i+1}\right)$ of $S_{k}^{i+1}$ under $x \rightarrow \beta_{i+1}(p, x)$ are disjoint as $p$ ranges over $A_{k}^{i+1}$ and $k$ varies from 1 to $n_{i+1}$; thus

$$
T_{k}^{i+1}=\bigcup_{p \in A_{k}^{i+1}} \beta_{i+1}\left(p, S_{k}^{i+1}\right)
$$

are disjoint, $1 \leq k \leq n_{i+1}$, and $\mu\left(T_{k}^{i+1}\right)=p_{k}^{i+1}$, so that $\bigcup_{k=1}^{n_{i+1}} T_{k}^{i+1}=X$.

We now define $\phi^{i+1}$. If $p, q$ and $p q \in A_{k}^{i+1}$, and $x \in S_{k}^{i+1}$, set

$$
\phi_{p}^{i+1}\left(\beta_{i+1}(q, x)\right)=\beta_{i+1}(p q, x) .
$$

Note that if $p, q$ and $p q \in A_{j}^{i+1}$, and $x \in S_{j}^{i+1}$,

$$
\phi_{p q}^{i+1}(x)=\beta_{i+1}(p q, x)=\phi_{p}^{i+1}\left(\beta_{i+1}(q, x)\right)=\phi_{p}^{i+1} \circ \phi_{q}^{i+1}(x),
$$

so that (iii) is satisfied. To extend the definition of $\phi^{i+1}$ to $\bigcup_{k=1}^{n_{i+1}} T_{k}^{i+1}$, let $R_{k}^{i+1}(p)$ be the set of points of $T_{k}^{i+1}$ at which $\phi_{p}^{i+1}$ is already defined. (Thus, if $p \notin A_{k}^{i+1}$, 
$\left.R_{k}^{i+1}(p)=\varnothing\right)$. Since

$$
\mu\left(\phi_{p}^{i}\left(T_{k}^{i+1}-R_{k}^{i+1}(p)\right)\right)=\mu\left(T_{k}^{i+1}-\phi_{p}^{i+1}\left(R_{k}^{i+1}(p)\right)\right)
$$

$-\phi_{p}^{i+1}\left(R_{k}^{i+1}(p)\right)$ being a subset of $T_{k}^{i+1}$-we may choose an $\mathscr{R}$-map $\phi_{p}^{i+1}$ from $\phi_{p}^{i}\left(T_{k}^{i+1}-R_{k}^{i+1}(p)\right)$ to $T_{k}^{i+1}-\phi_{p}^{i+1}\left(R_{k}^{i+1}(p)\right)$. We now clearly have $\phi_{p}^{i+1}$ a bijection of $T_{k}^{i+1}$ to itself for each $k, 1 \leq k \leq n_{i+1}$, and $\left(\phi_{p}^{i+1}(x), \phi_{p}^{i}(x)\right) \in \mathscr{R}$ for all $x \in X$.

Conditions (i), (ii), (iii) are now satisfied (for $i+1$ ); clearly (iv) is also satisfied. To check (v), suppose that $x \in S_{k}^{i+1}$ for some $k, p \in A_{j}^{i}$ for some $j, c \in C_{j, k}^{i}, q \in B_{j, k}^{i}(c)$ and that $p q \in A_{j}^{i}$ (so that $p, q, c, p q, q c, p q c$ all lie in $A_{k}^{i+1}$ ); then we have

$$
\begin{aligned}
\phi_{p}^{i} \circ \phi_{q c}^{i+1}(x) & =\phi_{p}^{i}\left(\beta_{i+1}(q c, x)\right)=\phi_{p}^{i} \circ \phi_{q}^{i} \circ \theta_{j, k}^{i}(c) \phi_{c}^{i}(x)=\phi_{p q}^{i+1}\left(\beta_{i+1}(c, x)\right) \\
& =\phi_{p}^{i+1} \circ \phi_{q}^{i+1}\left(\beta_{i+1}(c, x)\right)=\phi_{p}^{i+1}\left(\beta_{i+1}(q c, x)\right)=\phi_{p}^{i+1} \circ \phi_{q c}^{i+1}(x),
\end{aligned}
$$

so that $\phi_{p}^{i}=\phi_{p}^{i+1}$ on $\phi_{q c}^{i+1}\left(S_{k}^{i+1}\right)$. Now for $p \in A_{\ell}^{i-1}$,

$$
\left|\left\{q \in B_{j, k}^{i}(c): p q \in A_{j}^{i}\right\} /\right| A_{j}^{i} \mid>1-2 \varepsilon_{i},
$$

using the $\varepsilon_{i}$-invariance of $A_{j}^{i}$ under $A_{\ell}^{i-1}$ and the fact that $\left|B_{j, k}^{i}(c)\right| /\left|A_{j}^{i}\right|>1-\varepsilon_{i}$. The union of all the sets $\phi_{q c}^{i+1}\left(S_{k}^{i+1}\right)$ for $q, c$ as above thus has measure at least

$$
\left(1-2 \varepsilon_{i}\right) \sum_{k=1}^{n_{i+1}} \sum_{j=1}^{n_{i}}\left|A_{j}^{i}\right|\left|C_{j, k}^{i}\right| \mu\left(S_{k}^{i+1}\right)
$$

and this is at least

$$
\left(1-2 \varepsilon_{i}\right)\left(1-\varepsilon_{i}\right) \sum_{k}\left|A_{k}^{i+1}\right| \mu\left(S_{k}^{i+1}\right)=\left(1-2 \varepsilon_{i}\right)\left(1-\varepsilon_{i}\right) .
$$

Proof of Theorem 3.4. We first show that for any $p \in Q$, the sequence $\phi_{p}^{i}(x)$ stabilizes as $i \rightarrow \infty$ for almost all $x$. For $p \in A_{1}^{i}$, let

$$
G(i, p)=\left\{x \in X: \phi_{p}^{\ell}(x)=\phi_{p}^{\ell+1}(x) \text { for all } \ell \geq i+1\right\} ;
$$

note that by Lemma 3.13(v),

$$
\mu(G(\nu, p)) \geq 1-3 \sum_{\ell=i+1} \varepsilon_{i}
$$

so if $G(i)=\bigcap_{p \in A_{i}^{i}} G(i, p)$,

$$
\mu(G(i)) \geq 1-3\left|A_{1}^{i}\right| \sum_{\ell=i+1}^{\infty} \varepsilon_{i} .
$$

By choosing $\varepsilon_{i}$ to decrease sufficiently rapidly, we have $\mu(G(i))>1-\eta_{i}$, where $\eta_{i}$ decreases to zero as rapidly as we please. Thus $\mu(X-\lim \inf G(i))=0$. Since for any $p \in Q, p \in A_{1}^{i}$ eventually and since for $x \in \lim \inf G(i), \phi_{p}^{i+1}(x)$ is eventually constant, we are done.

Finally, we check multiplicativity. Recall from Lemma 3.13(iii), $\phi_{p q}^{i}=\phi_{p}^{i} \circ \phi_{q}^{i}$ on $S_{j}^{i}$ provided $p, q, p q \in A_{j}^{i}$. Thus if $p, q, r, p q, q r, p q r$ are all in $A_{j}^{i}$,

$$
\phi_{p q}^{i} \circ \phi_{r}^{i}(x)=\phi_{p q r}^{i}(x)=\phi_{p}^{i} \circ \phi_{q r}^{i}(x)=\phi_{p}^{i} \circ \phi_{q}^{i} \circ \phi_{r}^{i}(x) \text { for } x \in S_{j}^{i},
$$

and $\phi_{p q}^{i}=\phi_{p}^{i} \circ \phi_{q}^{i}$ on a set whose measure is at least $1-\eta_{i}$, where $\eta_{i} \rightarrow 0$ as rapidly as we please-this follows by an argument very similar to that used in the proof of Lemma 3.13(v), the details of which we leave to the reader. Arguing now as in the paragraph immediately above, we see that if $\psi_{p}=\lim _{i \rightarrow \infty} \phi_{p}^{i}, p \in Q$, then $\psi_{p} \circ \psi_{q}=\psi_{p q}$ for all $p, q \in Q$. 
We note that for groups $Q$ with property $T$ of Kazhdan, there is always at least one near action of $Q$ as automorphisms of an ergodic $\mathrm{II}_{1}$ relation which cannot be perturbed to be an action - an example may be built using the construction of [4] as a model, with essentially the same proof. Details are left to the reader. The situation for non-discrete groups $Q$ and discrete equivalence relations is completely unknown.

Again, we close with comments on the situation for discrete ergodic equivalence relations not of type $\mathrm{II}_{1}$. Theorems 3.1 and 3.2 remain valid when $\mathscr{R}$ is amenable and type $\mathrm{II}_{\infty}$, with no essential change in the proof, as does Corollary 3.3. However, the 'uniqueness' part of Theorem 3.2 changes - an additional invariant appears. Using the notation of Corollary 3.3, the homomorphism $\theta: Q \rightarrow$ Aut $(\mathscr{R})$ gives rise to a homomorphism mod $\theta: Q \rightarrow R$ defined by

$$
\bmod \theta(g)=\log \frac{d \mu \circ \theta(g)}{d \mu} .
$$

It is proven in [28] that $\bmod \theta$ is a complete invariant for the action of $Q$ on $R$ up to outer conjugacy, and therefore also for the pair $\mathscr{R} \subseteq \mathscr{R} \vee{ }_{\theta} Q$.

It is easy to see from Proposition 1.5 that Theorem 3.4 remains valid for any discrete group $Q$, amenable or not, and any properly infinite discrete ergodic equivalence relation $\mathscr{R}$. Again it is now possible to classify pairs $\mathscr{R} \subseteq \mathscr{S}$ with $\mathscr{S}$ amenable by applying further results of $[29,30]$.

\section{Rigidity}

Our concern here is to investigate pairs of equivalence relations $\mathscr{R} \subseteq \mathscr{S}$ under the assumption that $\mathscr{R}$ or $\mathscr{S}$ is generated by a free (or essentially free) II $_{1}$ ation of a lattice $\Gamma$ in a semi-simple Lie group, plus additional assumptions on the nature of the pair $\mathscr{R} \subseteq \mathscr{Y}$. The results are, for the most part, variations on a theme developed in [24 and the references therein], and we will draw heavily on results from these sources.

THEOREM 4.1. Let $G$ be a semi-simple non-compact connected Lie group with $\mathbb{R}$ rank $(G) \geq 2$, and the centre of $G$ being finite. Let $\Gamma \subseteq G$ be a lattice, let $(X, \mu)$ be any free ergodic $\mathrm{II}_{1} \Gamma$-space, let $\mathscr{S} \subseteq X \times X$ be the associated equivalence relation, and let $\mathscr{R} \subseteq \mathscr{S}$ be a normal ergodic subrelation. Then $\mathscr{R}$ has finite index in $\mathscr{S}$.

Proof. (First part.) We suppose first that $\mathscr{P} / \mathscr{R}=Q$ is amenable, and let $\theta: \mathscr{S} \rightarrow Q$ be the canonical cocycle with kernel $\mathscr{R}$. Since $\Gamma$ has Kazhdan's property $T, \theta$ is cohomologous to a cocycle $\theta_{0}$ taking values in a finite subgroup $Q_{0}$ of $Q,[24$, p. 162]. Since $\mathscr{S} \times{ }_{\theta} Q$ and hence $\mathscr{S} \times \theta_{\theta_{0}} Q$ is ergodic, $Q_{0}=Q$ is finite as required.

We will complete the proof by showing that $\mathscr{S} / \mathscr{R}$ must be amenable. This will be accomplished via the sequence of lemmas which follow. We keep the notation and hypothesis of the Theorem throughout our discussion.

We will need to work with induced actions, and begin by recalling the definition $[24$, p. 75] and some basic facts. If $(Z, \nu)$ is a (right) $\Gamma$-space, and $h: s \in \Gamma \backslash G \rightarrow G$ is a Borel section for the projection, we may define an action of $G$ on $(\tilde{Z}, \tilde{\nu})=$ $(\Gamma \backslash G \times Z, m \times \nu)$ where $m$ is the finite invariant measure on $\Gamma \backslash G$, by $(s, z) g=$ 
$\left(s g, z h(s) g h(s g)^{-1}\right)$. The $G$-space $(\tilde{Z}, \tilde{\nu})$ is called the $G$-space induced from the $\Gamma$-space $(Z, \nu)$. The map $p: \tilde{Z} \rightarrow \Gamma \backslash G$ defined by $p(s, z)=s$ is evidently Borel and $G$-equivariant. We record the following basic facts.

LemmA 4.2. Let $(Z, \nu),\left(Z_{1}, \nu_{1}\right)$ be $\Gamma$-spaces (where the action on $\left(Z_{1}, \nu_{1}\right)$ is the restriction to $\Gamma$ of a $G$ action $)$ and let $(\tilde{Z}, \tilde{\nu}),\left(\tilde{Z}_{1}, \tilde{\nu}_{1}\right)$ be the induced G-spaces, with $p, p_{1}$ the projections on $\Gamma \backslash G$. Then

(i) there is a G-equivariant isomorphism $\left(Z \times Z_{1}\right)^{\tilde{}} \rightarrow \tilde{Z} \times Z_{1}$ which commutes with the projections to $\Gamma \backslash G$. (Here $Z \times Z_{1}$ carries the diagonal action of $\Gamma$, and $\tilde{Z} \times Z_{1}$ the diagonal action of $G$.)

(ii) If there is a G-equivariant isomorphism $\phi:(\tilde{Z}, \tilde{\nu}) \rightarrow\left(\tilde{Z}_{1}, \tilde{\nu}_{1}\right)$ such that the diagram

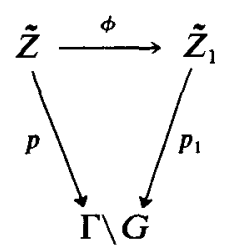

commutes, then, for almost all $s \in \Gamma$, the actions of the isotropy groups $G_{s}$ (of $G$ on $\Gamma \backslash G)$ at $s$ on $p^{-1}(s)$ and $p_{1}^{-1}(s)$ are conjugate.

Proof. (i) This is routine, and left to the reader.

(ii) This is the ergodic theory analogue of Mackey's Imprimitivity Theorem, $[15,23]$, and a proof may be constructed using the same technique; again, we leave details to the reader.

Lemma 4.3. Suppose $(Z, \nu)$ is an ergodic $\mathrm{II}_{1} \Gamma$-space, $\left(Z_{1}, \nu_{1}\right)$ is a $\Gamma$-space, and $P \subseteq G$ is a parabolic subgroup. Suppose there are measurable $\Gamma$-equivariant maps $f: Z \times$ $P \backslash G \rightarrow Z_{1}, g: Z_{1} \rightarrow Z$ with $g \circ f(z, P g)=z$. Then there is a parabolic subgroup $P_{1} \supseteq P$ of $G$ and $a \Gamma$-equivariant isomorphism $\phi$ of $Z_{1}$ with $Z \times P_{1} \backslash G$ so that

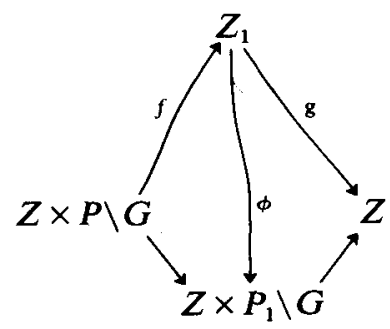

commutes, where the maps in the bottom line are the obvious ones.

Proof. Let $(Z \times P \backslash G) \tilde{Z}, \tilde{Z}_{1}, \tilde{Z}$ be the induced $G$-spaces, and let $\tilde{f}, \tilde{g}$ be the induced isomorphisms of $G$-spaces. By 4.2.1, we may identify $(Z \times P \backslash G)^{-}$with $\tilde{Z} \times P \backslash G$, and then obtain a $G$-equivariant diagram

$$
\tilde{\mathcal{Z}} \times P \backslash G \stackrel{\tilde{f}}{\longrightarrow} \tilde{\mathcal{Z}}_{1} \stackrel{\tilde{g}}{\longrightarrow} \tilde{\mathcal{Z}} .
$$

By $[24$, p. 150$]$, there is a parabolic subgroup $P_{1} \supseteq P$ and an $G$-equivariant iso- 
morphism $\tilde{\phi}$ from $\tilde{Z}_{1}$ to $\tilde{Z} \times P_{1} \backslash G$ such that the diagram

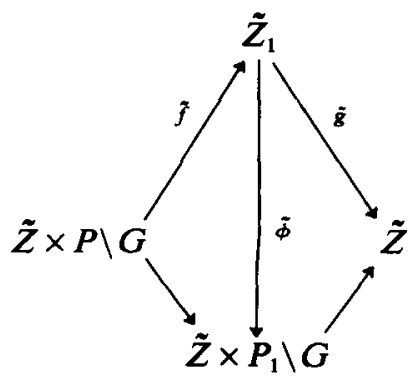

is $G$-equivariant and commutes, and the maps at the bottom are the obvious ones. Since all the maps in this last diagram commute with the canonical projections on $\Gamma \backslash G$ we may apply 4.2 (ii) to obtain the desired conclusion.

We note that Margulis [16] proves Lemma 4.3 in the case where $Z$ is a point. We will use the Lemma in a spirit very close to that of [16].

Proof of Theorem 4.1. (Final part.) Let $\mathscr{S} / \mathscr{R}=Q$ and $\theta: \mathscr{S} \rightarrow Q$ be as in the first part, and suppose $Q$ is not amenable. Let $A$ be a compact convex subset of a separable Banach space and $\pi: Q \rightarrow \operatorname{Aff}(A)$ be an affine action of $Q$ on $A$ with no fixed point. Thus $\pi \circ \theta: \mathscr{S} \rightarrow$ Aff $(A)$ is a cocycle; we let $P \subseteq G$ be a minimal parabolic and consider the cocycle $\beta:(X \times P \backslash G) \times \Gamma \rightarrow \operatorname{Aff}(A)$ which is the composition of $\pi \circ \alpha$ with the projection onto $X \times \Gamma=\mathscr{S}$. By [24, 4.3.4, 4.3.7] $X \times P \backslash G$ is an amenable $\Gamma$-space, so there exists a measurable map $\phi: X \times P \backslash G \rightarrow A$ such that $\beta((x, P g) \gamma) \circ \phi((x, P g) \gamma)=\phi(x, P g)$ for $\gamma \in \Gamma$ and $(x, P g) \in X \times P \backslash G$, or $\pi \circ \theta(x, \gamma)$ $(\phi(x, P g) \gamma)=\phi(x, P g)$.

Let $Z=X \times A$ with the skew product action $(x, a) \gamma=\left(x \gamma,(\pi \circ \theta(x, \gamma))^{-1}(a)\right)$ of $\Gamma$, define $\Phi: X \times P \backslash G \rightarrow Z$ by $\Phi(x, P g)=(x, \phi(x, P g))$ and let $\nu=(\mu \times m) \circ \Phi^{-1}$, where $m$ is a probability measure on $P \backslash G$ equivalent to the canonical quasi-invariant measure on $P \backslash G$. A routine calculation shows that $\Phi$ is $\Gamma$-equivariant; since the composition of $\Phi$ with the natural projection from $X \times A$ to $X$ is the projection of $X \times P \backslash G$ to $X$, we may apply Lemma 4.3 to conclude that as $\Gamma$-spaces, there is a parabolic subgroup $P_{1} \supseteq P$ and a $\Gamma$-equivariant isomorphism of $X \times P_{1} \backslash G$ with $Z$ such that

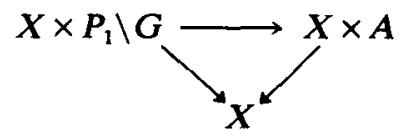

commute (the diagonal maps being projection).

We now claim $P_{1} \neq G$. If not, there is a $\Gamma$-invariant Borel map $\psi: X \rightarrow A$ i.e. $\psi$ satisfies $\pi \circ \theta(x, \gamma)^{-1} \psi(x)=\psi(x \gamma)$. But now $\psi(x)=\psi(x \gamma)$ for $(x, x \gamma) \in \mathscr{R}$, and since $\mathscr{R}$ is ergodic, $\psi(x)$ is a constant $a_{0} \in A$ for $\mu$-a.e. $x$; evidently, $a_{0}$ is fixed under $\pi(Q)$, contradicting the choice of $(A, \pi)$. We may thus assume $P_{1} \neq G$.

Let $U: G \rightarrow \mathcal{U}\left(L^{2}\left(P_{1} \backslash G\right)\right)=\mathcal{U}$ be the natural unitary representation of $G$ on $L^{2}\left(P_{1} \backslash G\right)$; we note that $U$ acts without fixed vectors, so that by [24, Theorem 2.2.20]. $U(G)$ is strongly closed in $\mathcal{U}$. We also note that as $P_{1} \neq G, U$ is faithful. 
Let $i: \mathscr{S}=X \times \Gamma \rightarrow \Gamma$ be the canonical cocycle, and $\theta \mid i_{G}$ be the composition of $i$ with the inclusion $\Gamma \subseteq G$. In view of the isomorphism of $X \times P_{1} \backslash G$ with the skew product $X \times_{\pi \circ \theta} A$ (as $\Gamma$-spaces), and in view of the triviality of $\left.\pi \circ \theta\right|_{\mathscr{R}^{\prime}}$ we conclude that the restriction of $U \circ i_{G}$ to $\mathscr{R}$ is trivial as a cocycle into $\mathscr{U}$. There is thus an $\mathscr{R}$-equivariant Borel map $u: X \rightarrow \mathcal{U}$ with $u(x)=U(\gamma) u(x \gamma)$ for $(x, x \gamma) \in \mathscr{R}$. The measure $\mu \circ u^{-1}$ is now ergodic for the action of $U(G)$ on $\mathcal{U}$ by left translation, and hence supported on a single orbit $U(G) u_{0}$, since $U / U(G)$ is countably separated, (see for example [8]). Write $u(x)=U(g(x)) u_{0}$ and note that we now have $U(g(x))=U\left(i_{G}(x, x y)\right) U(g(x y))$ for $(x, x y) \in \mathscr{R}$ so that $i_{G \mid \mathscr{R}}$ cobounds as a cocycle into $G$. Thus $g(x) g(x y)^{-1}=\gamma$ whenever $(x, x y) \in \mathscr{R}$. Choose a set $X_{0} \subseteq X$ of positive measure on which $g$ is bounded, so that for $x \in X_{0},\{\gamma \in \Gamma:(x, x y) \in \mathscr{R}, x \in$ $X_{0}$ and $x y \in X_{0}$ is also bounded. But this means that $\left.\mathscr{R}\right|_{X_{0}}$ has finite equivalence classes, contradicting the ergodicity of $\mathscr{R}$. The proof is now complete.

TheOREM 4.4. Let $G$ be a simple, connected, non-compact Lie group, and let $\Gamma \subseteq G$ be a lattice. Let $\mathscr{S}$ be the equivalence relation generated by a free $\mathrm{II}_{1}$ action of $\Gamma$ on $(X, \mu)$, and let $\mathscr{R} \subseteq \mathscr{S}$ be strongly normal and amenable. Then $\mathscr{R}$ is finite i.e. $\mathscr{R}(x)$ is finite for almost all $x$.

Before beginning the proof, we note that Theorem 4.5 has no rank assumptions on $G$, so the result applies to free groups. Further, Theorem 4.1 and 4.5 together constitute an analogue of Margulis' result that a normal subgroup of a (higher rank) lattice is either finite, or of finite index, [16].

Proof of 4.4. Let $i: \mathscr{S} \rightarrow \Gamma$ be the natural cocycle, and suppose that $\mathscr{R}$ is not finite. By Lemma 4.5 (see below), there is an algebraic, amenable non-central subgroup $A \subseteq G$ and an $\mathscr{R}$-invariant Borel map $\psi: X \rightarrow A \backslash G$ i.e. $\psi(x) i(x, \gamma)=\psi(x \gamma)$ for $(x, x y) \in \mathscr{R}$. Since $G$ is simple and $A$ is amenable, the normalizer $N_{G}\left(A^{0}\right)$ of $A^{0}$ in $G$ is not all of $G$. Since $N_{G}\left(A^{0}\right)$ is algebraic, we conclude by Lemma 4.6 (see below) the map $p \circ \psi: X \rightarrow N_{G}\left(A^{0}\right) \backslash G$ is $i$-invariant, and hence equivariant for the obvious $\Gamma$-actions. Since $X$ carries a finite $\Gamma$-invariant measure, there is a finite $\Gamma$-invariant measure $\nu$ on $N_{G}\left(A^{0}\right) \backslash G$. However, by $[24,83]$ the stabilizer $G_{\nu}$ of $\nu$ is algebraic; since $G_{\nu} \supseteq \Gamma$ and $\Gamma$ is Zariski dense, $G_{\nu}=G$. By the Borel density theorem [24, p. 41], $N_{G}\left(A^{0}\right)$ is discrete, so $A^{0}$ is trivial, and $N_{G}\left(A^{0}\right)=G$. This contradiction completes the proof modulo Lemmas $4.5,4.6$, which follow.

So suppose $\mathscr{R}$ is not finite. Since $\operatorname{Aut}_{\mathscr{P}}(\mathscr{R})$ generates $\mathscr{S}$ and $\mathscr{S}$ is ergodic, we conclude that the function $x \in X \rightarrow|\mathscr{R}(x)|$ (the cardinality of $\mathscr{R}(x)$ ) is constant a.e., and hence infinite. We conclude that $\mathscr{R}$ is in fact ergodic.

Lemma 4.5. Assume $\mathscr{R}$ is ergodic, and let $i: \mathscr{S} \rightarrow \Gamma$ be the canonical cocycle, let $i_{G}$ be the composition of $i$ with the inclusion into $G$, and let $A$ be the algebraic hull of $i_{G \mid \text { t }}$. Then

(a) $A$ is algebraic, amenable and non-central in $G$;

(b) there is $i_{G \mid \Re}$ invariant Borel map $\psi: x \rightarrow A \backslash G$ and any two such maps $\psi$ and $\psi^{\prime}$ satisfy $p \circ \psi=p \circ \psi^{\prime}$, where $p: A \backslash G \rightarrow N_{G}\left(A^{0}\right) \backslash G$ is the natural map. 
Proof. (a) The fact that the algebraic hull $A$ of $i_{G \mid \Re}$ is algebraic and amenable follows exactly as in [24, Theorem 9.2.3]. If $A$ is central, then the composition of $i_{G \mid \mathscr{R}}$ with the projection of $G$ onto $G / 3(G)(3(G)=$ the centre of $G)$ cobounds and the argument of the last paragraph of the proof of Theorem 4.1 shows that $i_{G \mid \Re}$ cobounds, and so that $\mathscr{R}$ is finite. Thus $A$ is non-central.

(b) follows from the arguments of $[24$, p. 174, last paragraph] without change.

Lemma 4.6. Let $i, A, \psi$ be as in Lemma 4.6. Then $p \circ \psi$ is invariant under $i$.

Proof. Let $\phi \in \operatorname{Aut}_{\mathscr{P}}(\mathscr{R})$, and define $\psi^{\phi}: X \rightarrow A \backslash G$ by $\psi^{\phi}(x)=\psi(\phi x) i(\phi x, x)$. Note that if $\left(x, x^{\prime}\right) \in \mathscr{R}$,

$$
\begin{aligned}
\psi^{\phi}\left(x^{\prime}\right) i\left(x^{\prime}, x\right) & =\psi\left(\phi x^{\prime}\right) i\left(\phi x^{\prime}, x^{\prime}\right) i\left(x^{\prime}, x\right) \\
& =\psi\left(\phi x^{\prime}\right) i\left(\phi x^{\prime}, \phi x\right) i(\phi x, x) \\
& =\psi(\phi x) i(\phi x, x)=\psi^{\phi}(x),
\end{aligned}
$$

so that $\psi^{\phi}$ is also $i_{G \mid \Re}$ invariant. From 4.5(b) we conclude $p \circ \psi=p \circ \psi^{\phi}$, so that $p \circ \psi$ is invariant under the restriction of $i_{G}$ to the equivalence relation generated by $\mathscr{R}$ and $\phi$. Since $\mathscr{S}$ is generated by $\mathscr{R}$ and a countable set of elements from Aut $_{\mathscr{S}}(\mathscr{R}), p \circ \psi$ is in fact $i_{G}$ invariant as required.

We remark that the only essential use of the hypothesis of strong normality of $\mathscr{R}$ in $\mathscr{S}$ is in the discussion immediately preceding Lemma 4.5 , since the argument in Lemma 4.6 works equally well for endomorphisms $\phi \in \operatorname{End}_{\mathscr{S}}(\mathscr{R})$. The conclusion of Theorem 4.5 is thus valid for normal subrelations $\mathscr{R}$ of $\mathscr{S}$ provided $|\mathscr{R}(x)|$ is a.e. constant.

There is another situation in which the conclusions of Theorem 4.5 remain valid.

THEOREM 4.8. Let $N$ be a complete finite volume Riemannian manifold with sectional curvature $k \leq c<0$, where $c$ is fixed, and let $\Gamma \subset \pi_{1}(N)$. Then if $\Gamma$ is not amenable, the conclusions of Theorem 4.5 are valid for $\Gamma$.

Proof. We give a sketch only, since the ideas involved are already contained in [27] and the proof of Theorem 4.5.

Let $\tilde{N}$ be the universal cover of $N$, let $\partial \tilde{N}$ be boundary of $N$ as in $[6,27]$, and consider the natural action of $\Gamma$ on $\tilde{N}$ by deck transformations, and on $\partial \tilde{N}$ as in [6]. Recall that the stabilizer of any one or two-point set in $\partial \tilde{N}$ is amenable, [7, Corollary 3.3]. In addition, if $M(\partial \tilde{N})$ is the space of probability measures on $\partial \tilde{N}$, and $\gamma_{n} \in \Gamma$ is a sequence with $\gamma_{r} \rightarrow \infty$, there is a one or two-point set $S=S\left(\left\{\gamma_{n}\right\}\right) \subseteq$ $\partial \tilde{N}$ such that if $\gamma_{n} \nu \rightarrow \nu_{0}$ for some $\nu, \nu_{0} \in M(\partial \tilde{N})$, then $\operatorname{supp}\left(\nu_{0}\right) \subseteq S ; S$ is uniquely determined by $\left\{\gamma_{n}\right\}$ - see [27, Theorem 3.7].

Suppose now $\mathscr{R} \subseteq \mathscr{S}$ is as in the statement of Theorem 4.8, and $\mathscr{R}$ is not finite. As in the proof of Theorem 4.5, we may suppose $\mathscr{R}$ is ergodic. Let $i: \mathscr{S} \rightarrow \Gamma$ be the canonical cocycle, and note that since $\mathscr{R}$ is amenable, there is an $\left.i\right|_{\mathscr{R}}$-invariant function $\nu$ from $X$ to $M(\partial \tilde{N})$. Arguing as in the proof of Lemma 5.2 of [27], $\operatorname{supp}\left(\nu_{x}\right)$ is a one or two-point set $S(x)$ for almost all $x$, and $S(x)$ is uniquely determined. By perturbing $\nu$ by elements of Aut $\operatorname{s}_{\mathscr{S}}(\mathscr{R})$ as was done to $\phi$ in the proof of Lemma 4.6, we conclude that $x \rightarrow \nu(x) \in M(\partial \tilde{N})$ is in fact $i$-invariant (on all of 
$\mathscr{S})$. Transporting the $\Gamma$-invariant measure $\mu$ to $\partial \tilde{N}$, i.e. considering $\nu_{0}=$ $\int^{\oplus} \nu(x) d \mu(x)$, we deduce the existence of a $\Gamma$-invariant measure $\nu_{0}$ on $\partial \tilde{N}$. Now $\nu_{0}$ has support in a one or two point set, and we conclude that $\Gamma$ is amenable. This contradiction completes the proof.

We turn now to our final rigidity type result. As shown in [24, Corollary 5.2.2], lattices in distinct connected simple Lie groups (of rank at least two) can never have orbit equivalent free ergodic $\mathrm{II}_{1}$-actions. We shall prove that such equivalence relations remain distinct even when enlarged by an outer action of a discrete amenable torsion free group. The torsion-free hypothesis is almost certainly not necessary, but at this point, we do not see how to remove it.

We begin with some generalities. Let $G$ be a connected simple Lie group with trivial centre and with rank $G \geq 2$, let $\Gamma \subseteq G$ be a lattice, and let $\mathscr{R}$ be the equivalence relation generated by a free ergodic $\mathrm{II}_{1} \Gamma$-action on $(X, \mu)$. Let $\mathscr{S}$ be a $\mathrm{II}_{1}$-equivalence relation on $(X, \mu)$ with $\mathscr{R}$ normal in $\mathscr{S}$, and suppose $\mathscr{S} / \mathscr{R}=A$. We let $\left\{\phi_{a}: a \in A\right\} \subseteq$ Aut $_{\mathscr{S}}(\mathscr{R})$ be automorphisms with $\phi_{a} \phi_{b}=\phi_{a b}$ modulo Int $\mathscr{R}$ and $\mathscr{R} \vee_{\phi} A=\mathscr{S}$.

Let $(\tilde{X}, \tilde{\mu})$ be the induced $G$-space, and let $\tilde{\mathscr{R}}$ denote the equivalence relation generated by the $G$-action. As noted in $[24$, p. 75], $\tilde{R}$ is isomorphic with $\mathscr{R} \times \mathscr{T}(\Gamma \backslash G)$, where $\mathscr{T}(\Gamma \backslash G)$ is the transitive relation on $\Gamma \backslash G$. Evidently, $\tilde{\phi}_{a}=\phi_{a} \times$ id are now automorphisms of $\tilde{\mathscr{R}}$, and $\tilde{\mathscr{R}} \vee_{\tilde{\phi}} A=\tilde{\mathscr{T}}=\mathscr{S} \times \mathscr{T}(\Gamma \backslash G)$.

Proposition 4.9. With $\left\{\tilde{\phi}_{a}: a \in A\right\}$ as above, there exist homomorphisms $a \in A \rightarrow \tilde{\psi}_{a} \in$ Aut (R), $a \in A \rightarrow \gamma_{a} \in$ Aut $(G)$ such that

(i) $\left(\tilde{\psi}_{a}(y), \tilde{\phi}_{a}(y)\right) \in \tilde{\mathscr{R}}$ for each $a \in A$ and $y \in \tilde{X}$;

(ii) $\tilde{\psi}_{a} g \tilde{\psi}_{a}^{-1}=\gamma_{a}(g), g \in G$.

Furthermore, $\gamma_{a}$ is uniquely determined up to inner automorphisms of $G$.

Proof. The proof of [24, Theorem 5.2.1] guarantees for each $a \in A$ the existence of automorphisms $\tilde{\psi}_{a}, \gamma_{a}$ satisfying (i) and (ii). We first prove uniqueness of $\gamma_{a}$ up to Int $(G)$. Evidently it is enough to show that if $\tilde{\psi} \in \operatorname{Int}(\tilde{R})$ and $\tilde{\psi} g \tilde{\psi}^{-1}=\kappa(g)$ for some $\kappa \in$ Aut $(G)$ then $\kappa$ is inner.

Write $\tilde{\phi}(y)=y h(y)$ for $y \in \tilde{X}$, where $h: \tilde{X} \rightarrow G$ is Borel. Since $\tilde{\phi} g \tilde{\phi}^{-1}=\kappa(g)$ we obtain $y g h(y g)=y h(y) \kappa(g)$ a.e. and hence $g h(y g)=h(y) \kappa(g)$ or $h(y g)=$ $g^{-1} h(y) \kappa(g)$. Thus $h: \tilde{X} \rightarrow H$ is equivariant for the given action of $G$ on $\tilde{X}$ and the action $g_{1} \rightarrow g^{-1} g_{1} \kappa(g)$ of $G$ on itself. Since $G$ is algebraic, and $\kappa$ is automatically algebraic (see [2]), this last action is smooth ([24, p. 33]). Thus $\mu \circ h^{-1}$ is concentrated on an orbit $G / G_{0}$, where $G_{0}$ is the stabilizer of a point $g_{0} \in G$. Now

$$
\begin{aligned}
G_{0} & =\left\{g \in G: g^{-1} g_{0} \kappa(g)=g_{0}\right\} \\
& =\left\{g \in G: \kappa(g)=g_{0}^{-1} g g_{0}\right\},
\end{aligned}
$$

which is an algebraic subgroup. But $G / G_{0}$ supports a finite invariant measure $\mu \circ h^{-1}$, so by the Borel density Theorem ([24, p. 41]), $G_{0}=G$, and $\kappa(g)=g_{0}^{-1} g g_{0}$ for all $g$, as required.

Thus, if $\varepsilon$ : Aut $(G) \rightarrow$ Aut $(G) / \operatorname{Int}(G)$ is the quotient homomorphism, $\varepsilon \circ \gamma$ is a homomorphism. By [2], $\varepsilon$ is split, so that modifying $\tilde{\psi}_{a}$ if necessary, we may assume that $y$ is a homomorphism. 
To complete the proof, note that if $\tilde{\psi}$ is in Int $(\mathscr{R})$ and $\tilde{\psi} g \tilde{\psi}^{-1}=\mathbf{g}$ on $\tilde{X}$ for each $g$ then, writing $\tilde{\psi}(y)=y h(y)$ with $h: \tilde{X} \rightarrow G$ Borel, we obtain $h(y g)=g^{-1} h(y) g$. As above $\mu \circ h^{-1}$ is supported on the orbit of some point $g_{0} \in G$, so that $G / C_{G}\left(g_{0}\right)$ carries a finite invariant measure, $C_{G}\left(g_{0}\right)$ being the centralizer of $g_{0}$ in $G$. Using Borel density again, $C_{G}\left(g_{0}\right)=G$, so $g_{0}$, being central, is the identity, and $h(y)$ is the identity for all $y$. We conclude $\tilde{\psi}$ is the identity. It now follows that if $\left(\tilde{\psi}_{a}, \gamma_{a}\right)$ satisfy (i) and (ii) of Proposition 4.9, and if $\gamma$ is a homomorphism, so is $\tilde{\psi}$.

Let $\gamma$ and $\tilde{\psi}$ be as in Proposition 4.9, and consider the semi-direct product $G \times{ }_{\gamma} A$. We note the map

$$
(g, a) \in G \times{ }_{\gamma} A \rightarrow g \tilde{\psi}_{a} \in \operatorname{Aut}(\tilde{X}, \tilde{\mu})
$$

is now a representation of $G \times_{\gamma} A$ on $(\tilde{X}, \tilde{\mu})$ which generates $\tilde{\mathscr{S}}$, with the $G$ action generating $\tilde{\mathscr{R}}$.

THEOREM 4.10. For $j=1,2$, let $G_{j}$ be simple connected centre-free Lie groups with rank $G_{j} \geq 2$, let $\Gamma_{j} \subseteq G_{j}$ be lattices, and let $\mathscr{R}_{j}$ be the equivalence relations generated by free ergodic $\mathrm{II}_{1}$-actions of $\Gamma_{j}$ on $\left(X_{j}, \mu_{j}\right)$. Let $\mathscr{S}_{j}$ be $\mathrm{II}_{1}$-equivalence relations with $\mathscr{R}_{j}$ normal in $\mathscr{S}_{j}$, and $\mathscr{S}_{j} / \mathscr{R}_{j}=A_{j}$ amenable and torsion free. Then, if $\mathscr{S}_{1}$ and $\mathscr{Y}_{2}$ are isomorphic, $G_{1}$ is isomorphic with $G_{2}$.

Proof. Let $\tilde{\mathscr{R}}_{j}, \tilde{\mathscr{S}}_{j}$ be the induced equivalence relations on $\left(\tilde{X}_{j}, \tilde{\mu}_{j}\right)$, and let $\gamma_{j}: A_{j} \rightarrow$ Aut $\left(G_{j}\right)$ be as provided by Proposition 4.9. If $\mathscr{S}_{1}$ and $\mathscr{S}_{2}$ are orbit equivalent, so are $\tilde{\mathscr{S}}_{1}$ and $\tilde{\mathscr{S}}_{2}$. Let $\tilde{\phi}$ be such an orbit equivalence and let $\alpha \in Z^{1}\left(\tilde{\mathscr{S}}_{1}, G_{2} \times{ }_{\gamma_{2}} A_{2}\right)$ be the associated cocycle. If $\pi_{2}$ is the projection of $G_{2} \times_{\gamma_{2}} A_{2}$ on $A_{2}, \pi_{2} \circ \alpha \in$ $Z^{1}\left(\tilde{\mathscr{F}}_{1}, A_{2}\right)$; since $A_{2}$ is torsion free, $\left.\pi_{2}{ }^{\circ} \alpha\right|_{\mathscr{R}_{1}}$ cobounds by [24, p. 162]. We may thus assume that $\left.\alpha\right|_{\pi_{1}}$ takes values in $G_{2}$ and that there is a map $\tilde{\phi}_{1}: \tilde{X}_{1} \rightarrow \tilde{X}_{2}$ with $\tilde{\theta}_{1}(y g)=\tilde{\theta}_{1}(y) \alpha(y, y g)$ for $(y, g) \in \tilde{X}_{1} \times G_{1}$, and with $\tilde{\theta}_{1}(y)$ lying on the $A_{2}$-orbit of $\tilde{\theta}(y)$ for almost all $y \in \tilde{X}_{1}$. Note $\tilde{\mu}_{2}\left(\tilde{\theta}_{1}\left(\tilde{X}_{1}\right)\right)>0$, so we conclude that $\tilde{\theta}_{1}\left(\tilde{R}_{1}(y)\right) \subseteq$ $\tilde{R}_{2}\left(\tilde{\theta}_{1}(y)\right)$ for almost all $y \in \tilde{X}_{1}$.

Let $C_{2} \subseteq G_{2}$ be the algebraic hull of $\left.\alpha\right|_{\mathscr{R}_{1}}$ (see [24, p. 166]) and let $N_{2} \subseteq C_{2}$ be the maximal normal amenable subgroup. We may assume that $\left.\alpha\right|_{\tilde{R}_{1}}$ takes values in $C_{2}$. Note that if $N_{2}=C_{2}, C_{2}$ is amenable, so by [24, p. 162], $\alpha$ cobounds into a compact subgroup $K_{2}$ of $C_{2}$. There is thus a Borel map $\tilde{\theta}_{1}^{\prime}: \tilde{X}_{1} \rightarrow \tilde{X}_{2}$ with

$$
\tilde{\mathscr{R}}_{2}\left(\tilde{\theta}_{1}^{\prime}(y)\right)=\tilde{\mathscr{R}}_{2}\left(\tilde{\theta}_{1}(y)\right) \text { a.e. }
$$

and

$$
\tilde{\theta}_{1}^{\prime}\left(\tilde{R}_{1}(y)\right) \in \tilde{\theta}_{1}^{\prime}(y) K_{2} \quad \text { a.e. }
$$

But $K_{2}$ acts smoothly, so for some fixed $y_{2} \in \tilde{X}_{2}, \tilde{\theta}_{1}^{\prime}(y) \in y_{2} K_{2}$ for almost all $y \in \tilde{X}_{1}$. But then $\tilde{\theta}_{1}(y) \in y_{2} G_{2}$ for almost all $y \in \tilde{X}_{2}$ which is a contradiction. We may thus assume $C_{2} / N_{2}$ is non-trivial.

Since $C_{2} / N_{2}$ has no compact subgroup, the cocycle super-rigidity theorem of [24, p. 98] guarantees the existence of a non-trivial rational homomorphism $\pi_{1}^{\prime}: G_{1} \rightarrow$ $C_{2} / N_{2}$. However, there is a local splitting from $C_{2} / N_{2}$ to $C_{2}$, so there is a non-trivial local homomorphism $\pi_{1}: G_{1} \rightarrow G_{2}$.

Reversing the roles of $\tilde{\mathscr{F}}_{1}$ and $\tilde{\mathscr{F}}_{2}$, we similarly obtain a non-trivial local rational homomorphism $\pi_{2}: G_{2} \rightarrow G_{1}$, and hence isomorphism of $G_{1}$ and $G_{2}$. 


\section{Unsolved problems}

We would like to conclude by mentioning some unsolved problems which merit consideration.

The first and simplest is in regard to Theorem 4.10; the hypothesis that $A_{j}$ is torsion free should not be necessary $(j=1,2)$, but we do not know how to remove it.

The second is in relation to Theorem 2.13, vihich establishes a lattice isomorphism between intermediate relations $\mathscr{T}, \mathscr{R} \subseteq \mathscr{T} \subseteq \mathscr{S}$, and subgroups of the quotient $\mathscr{S} / \mathscr{R}=$ $Q$ (when $\mathscr{R}$ is normal and ergodic in $\mathscr{S}$ ). If $\mathscr{R}$ is amenable, and $N$ is the maximal normal amenable subgroup in $Q$, it is routine to check that $\mathscr{T}_{N}$ is a maximal, normal, amenable subrelation of $\mathscr{S}$. We know of examples of relations $\mathscr{S}$ which contain distinct maximal normal amenable subrelations, but we know of no examples of relations $\mathscr{S}$ admitting maximal normal amenable subrelations which are not conjugate under Aut $(\mathscr{P})$. On the other hand, Theorem 4.4 and 4.8 give examples of equivalence relations which admit no amenable, ergodic, normal subrelations. We are thus faced with two problems: determine when a given equivalence relation admits a normal amenable ergodic subrelation, and determine under what conditions maximal such subrelations are unique up to conjugacy.

The final problem concerns what might be called the 'algebra of normal subrelations'. Thus for example, if $\mathscr{R}, \mathscr{T}$ are normal and ergodic in $\mathscr{S}$, we should have $\mathscr{R} \vee \mathscr{T} / \mathscr{R}$ isomorphic with $\mathscr{S} / \mathscr{R} \cap \mathscr{T}$, and this is indeed true if $\mathscr{R} \cap \mathscr{T}$ is ergodic. It would be desirable to find a correct version of this statement when $\mathscr{R} \cap \mathscr{I}$ is not ergodic, and to find analogues of the other standard computational tools for normal subgroups from group theory.

\section{REFERENCES}

[1] R. G. Brown. Fibrations of groupoids. Journal of Algebra 15 (1) (1970), 103-132.

[2] C. Chevalley. Theory of Lie Groups (Princeton University Press: Princeton, 1946).

[3] A. Connes, J. Feldman \& B. Weiss. An amenable equivalence relation is generated by a single transformation. Ergod. Th. \& Dynam. Sys. I (1981), 431-450.

[4] A. Connes \& V. Jones. Property T for von Neumann Algebras. Bull. Lon. Math. Soc. 17 (1985), 57-62.

[5] H. A. Dye. On groups of measure preserving transformations I and II. Amer. J. Math. 81 (1959), 119-159 and Amer. J. Math. 85 (1963), 551-576.

[6] P. Eberlein. Geodesic flows on negatively curved manifolds I, II. Ann. Math. 95 (1972), 492-510 and T.A.M.S. 1978 (1973), 57-82.

[7] P. Eberlein. Lattices in spaces of non-positive curvature. Ann. Math. 111 (1980), 455-467.

[8] E. Effros. Transformation groups and $C^{*}$-algebras. Ann. Math. 81 (1965), 38-55.

[9] R. C. Fabec. Normal Ergodic actions and Extensions. Israel J. Math. 40 (2) (1981), 175-186.

[10] J. Feldman \& C. C. Moore. Ergodic equivalence relations, cohomology and von Neumann algebras I and II. T.A.M.S. 234 (1977) 289-324 and 325-359.

[11] M. Gerber. Classifying the finite extensions of an ergodic transformation up to factor orbit equivalence. Preprint.

[12] V. Ya. Golodets \& S. I. Sinelchikov. Existence and uniqueness of cocycles of an ergodic automorphism with dense ranges in amenable groups. Preprint 19-83, FTINT AN USSR, Kharkov, 1-21.

[13] V. F. R. Jones. Index for subfactors. Inventiones Math. 72 (1983), 1-25.

[14] G. W. Mackey. Borel spaces for groups and their duals. T.A.M.S. 85 (1957), 134-165.

[15] G. W. Mackey. Infinite dimensional group representations. B.A.M.S. 69 (1963), 628-686. 
[16] G. A. Margulis. Finiteness of quotient groups of discrete subgroups. Funct. Anal. Appl. 13 (1979), 178-187.

[17] A. Ocneanu. Actions of discrete amenable groups on von Neumann algebras. Springer Lecture Notes in Math. 1138 (Springer-Verlag: New York, 1985).

[18] D. Ornstein \& B. Weiss. Ergodic theory of amenable group actions. Preprint.

[19] A. Ramsay. Virtual groups and group actions. Adv. Math. 6 (1971), 253-322.

[20] K. Schmidt. Strong ergodicity and quotients of equivalence relations. To appear in Proc. of the Centre for Mathematical Analysis (Australian National University, Canberra).

[21] C. E. Sutherland. Sub-equivalence relations and subalgebras of von Neumann algebras. In preparation.

[22] C. E. Sutherland. A Borel parametrization of Polish groups. R.I.M.S. Kyoto University 21 (6) (1985), $1067-1086$

[23] V. S. Varadarajan. The Geometry of Quantum Theory. Vol II (van Nostrand Reinhold, New York, 1970).

[24] R. J. Zimmer. Ergodic Theory and Semi-simple groups (Birkhauser, 1984).

[25] R. J. Zimmer. Extensions of ergodic group actions. Illinois J. Math. 20 (1976), 373-409.

[26] R. J. Zimmer. Normal ergodic actions. J.F.A. 25 (3) (1977), 281-305.

[27] R. J. Zimmer. Ergodic Theory, Semi-simple Groups and foliations by manifolds of negative curvature. Pub. Math. I.H.E.S. 55 (1982), 37-61.

[28] S. I. Bezuglyi \& V. Ya. Golodets. Outer conjugation of actions of countable groups on a measure space. Preprint 2-84, F.T.I.N.T. AN U.S.S.R., Kharkov.

[29] S. I. Bezuglyi \& V. Ya. Golodets. Groups of measure space transformations and invariants of outer conjugation for automorphisms from normalizers of type III full groups. J.F.A. 60 (1985), 341-369.

[30] S. I. Bezuglyi \& V. Ya. Golodets. Measure space transformations and outer conjugacy of countable amenable automorphism groups. Preprint 28-85, F.T.I.N.T. AN, U.S.S.R., Kharkov. 